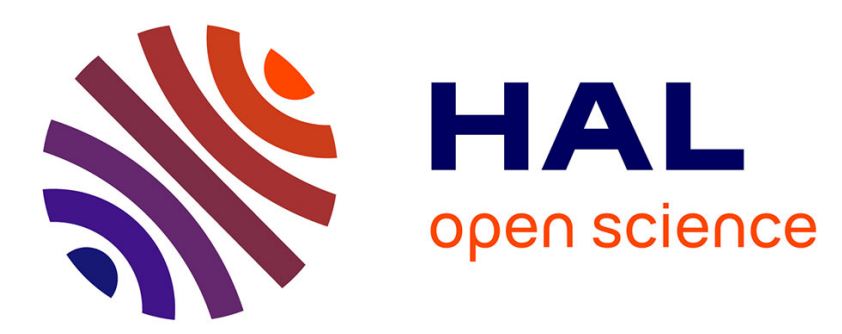

\title{
Experimental investigation of flow-induced vibration of a rotating circular cylinder
}

\author{
K.W.L. Wong, Jisheng Zhao, David Lo Jacono, Mark C. Thompson, John \\ Sheridan
}

\section{> To cite this version:}

K.W.L. Wong, Jisheng Zhao, David Lo Jacono, Mark C. Thompson, John Sheridan. Experimental investigation of flow-induced vibration of a rotating circular cylinder. Journal of Fluid Mechanics, 2017, 829, pp.486-511. 10.1017/jfm.2017.540 . hal-01619818

\section{HAL Id: hal-01619818 https://hal.science/hal-01619818}

Submitted on 19 Oct 2017

HAL is a multi-disciplinary open access archive for the deposit and dissemination of scientific research documents, whether they are published or not. The documents may come from teaching and research institutions in France or abroad, or from public or private research centers.
L'archive ouverte pluridisciplinaire HAL, est destinée au dépôt et à la diffusion de documents scientifiques de niveau recherche, publiés ou non, émanant des établissements d'enseignement et de recherche français ou étrangers, des laboratoires publics ou privés. 


\section{OATAO \\ Open Archive Toulouse Archive Ouverte}

\section{Open Archive TOULOUSE Archive Ouverte (OATAO)}

OATAO is an open access repository that collects the work of Toulouse researchers and makes it freely available over the web where possible.

This is an author-deposited version published in : http://oatao.univ-toulouse.fr/ Eprints ID : 18605

To link to this article : DOI: $10.1017 / \mathrm{jm} .2017 .540$

URL : http://dx.doi.org/10.1017/jfm.2017.540

To cite this version : Wong, K.W.L. and Zhao, Jisheng and Lo Jacono, David and Thompson, Mark C. and Sheridan, John Experimental investigation of flow-induced vibration of a rotating circular cylinder. (2017) Journal of Fluid Mechanics, vol. 829. pp. 486-511. ISSN 00221120

Any correspondence concerning this service should be sent to the repository administrator: staff-oatao@listes-diff.inp-toulouse.fr 


\title{
Experimental investigation of flow-induced vibration of a rotating circular cylinder
}

\author{
K. W. L. Wong ${ }^{1}$, J. Zhao ${ }^{1,} \dagger$, D. Lo Jacono ${ }^{1,2}$, M. C. Thompson ${ }^{1}$ \\ and J. Sheridan ${ }^{1}$ \\ ${ }^{1}$ Fluids Laboratory for Aeronautical and Industrial Research (FLAIR), Department of Mechanical \\ and Aerospace Engineering, Monash University, Melbourne, VIC 3800, Australia \\ ${ }^{2}$ Institut de Mécanique des Fluides de Toulouse (IMFT), Université de Toulouse, CNRS, INPT, UPS, \\ 31400 Toulouse, France
}

\begin{abstract}
While flow-induced vibration of bluff bodies has been extensively studied over the last half-century, only limited attention has been given to flow-induced vibration of elastically mounted rotating cylinders. Since recent low-Reynolds-number numerical work suggests that rotation can enhance or suppress the natural oscillatory response, the former could find applications in energy harvesting and the latter in vibration control. The present experimental investigation characterises the dynamic response and wake structure of a rotating circular cylinder undergoing vortex-induced vibration at a low mass ratio $\left(\mathrm{m}^{*}=\right.$ $5.78)$ over the reduced velocity range leading to strong oscillations. The experiments were conducted in a free-surface water channel with the cylinder vertically mounted and attached to a motor that provided constant rotation. Springs and an air-bearing system allow the cylinder to undertake low-damped transverse oscillations. Under cylinder rotation, the normalised frequency response was found to be comparable to that of a freely vibrating non-rotating cylinder. At reduced velocities consistent with the upper branch of a non-rotating transversely oscillating cylinder, the maximum oscillation amplitude increased with non-dimensional rotation rate up to $\alpha \approx 2$. Beyond this, there was a sharp decrease in amplitude. Notably, this critical value corresponds approximately to the rotation rate at which vortex shedding ceases for a non-oscillating rotating cylinder. Remarkably, at $\alpha=2$ there was approximately an $80 \%$ increase in the peak amplitude response compared to that of a non-rotating cylinder. The observed amplitude response measured over

the Reynolds-number range of $(1100 \lesssim R e \lesssim 6300)$ is significantly different from numerical predictions and other experimental results recorded at significantly lower Reynolds numbers.
\end{abstract}

Key words: flow-structure interactions, vortex streets, wakes

\section{Introduction}

Flow-induced vibration (FIV) of elastically mounted bluff bodies is observed in applications as diverse as the oscillation of a flagpole in the wind, the vibration of

$\dagger$ Email address for correspondence: jisheng.zhao@monash.edu 
cooling arrays in nuclear power generators and offshore oil risers. These vibrations can decrease the life of engineering structures and even cause catastrophic failures. Design methods used to avert such effects require a means of predicting their occurrence and characteristics. This has motivated numerous investigations that aim to characterise the fluid-structure system and provide a good understanding of the mechanisms. Surveys of the field are covered in reviews by Griffin, Skop \& Koopmann (1973), Bearman (1984), Blevins (1990), Carberry, Sheridan \& Rockwell (2001), Sarpkaya (2004), Williamson \& Govardhan (2004), Naudascher \& Rockwell (2005) and Païdoussis, Price \& De Langre (2010), amongst others. FIV, on the other hand, could also find applications in energy harvesting and vibration control. Comprehensive reviews on this topic are given by Xiao \& Zhu (2014) and Young, Lai \& Platzer (2014).

There are two body oscillator phenomena typical of FIV: vortex-induced vibration (VIV) and transverse galloping (see Naudascher \& Rockwell 2005). VIV is caused by vortex shedding in the wake of a body that then causes structural vibration due to the spatially oscillating pressure field associated with the shed vortices. Since the pioneering study by Feng (1968), VIV has been extensively investigated using the canonical model of an elastically mounted rigid circular cylinder that is free or forced to oscillate transversely in a free stream (Bishop \& Hassan 1964; Bearman 1984, 2011; Williamson \& Roshko 1988; Govardhan \& Williamson 2000; Carberry et al. 2001; Morse \& Williamson 2009; Zhao et al. 2014a).

While the axial symmetry of a circular cross-section enables VIV to be studied independently from other forms of FIV (i.e. galloping), it should be noted that VIV can still occur under certain conditions (e.g. mass and damping ratios) and dominate over a range of reduced velocity when the axial symmetry is broken, as demonstrated by Corless \& Parkinson (1988), Nemes et al. (2012) and Zhao et al. (2014b). In such cases, however, the body may also be subjected to transverse galloping. Galloping is categorised as movement-induced excitation (MIE) by Naudascher \& Rockwell (2005). It commonly occurs when the cylindrical body is not axisymmetric, making it aerodynamically unstable to transverse oscillations (Blevins 1990; Naudascher \& Rockwell 2005; Païdoussis et al. 2010). Generally, galloping is characterised by oscillations with amplitudes increasing monotonically with flow speed and frequencies much lower than that of vortex shedding (Bearman et al. 1987); for instance, iced electrical transmission cables were observed to oscillate with low frequencies and amplitudes of the order of 100 diameters in winds (see Den Hartog 1932). The galloping response is attributable to the asymmetric pressure distribution over the body that is created by the changing instantaneous angle of attack between the flow and the body. VIV and galloping need not occur mutually exclusively; their occurrence depends on the flow and structural properties. In fact, the two FIV phenomena may occur in the same flow regimes, as shown in Corless \& Parkinson (1988), Nemes et al. (2012) and Zhao et al. (2014b). Nemes et al. (2012) and Zhao et al. (2014b) have shown that large amplitude response regions can result from the interaction between VIV and galloping.

The present study introduces asymmetry into the system via actuating the body through the application of forced rotation to an elastically mounted circular cylinder. Similar mechanical configurations could have practical applications in offshore engineering, where long slender rotating shafts are exposed to varying flow in an ocean current. Understanding the behaviour of a rotating cylinder undergoing VIV may enable behavioural prediction of similar mechanical systems, and could contribute to energy harvesting applications and FIV control, given the possibility of enhancing or suppressing the vibrations. 
Rigidly mounted (non-oscillating) circular cylinders undergoing forced rotation in a cross-flow $h$ ave a lso $b$ een $t$ he $s$ ubject of $s$ tudies a nd $r$ eviews o ver the $\mathrm{p}$ ast century (e.g. Tietjens \& Prandtl 1957; Swanson 1961; Coutanceau \& Ménard 1985; Mittal \& Kumar 2003; Rao et al. 2013), due to the potential for wake manipulation. Different wake regimes have been identified in these $\mathrm{p}$ revious s tudies that a re found to depend on the non-dimensional rotation rate of the cylinder, defined a $\mathrm{s} t$ he $\mathrm{r}$ atio between the cylinder surface $(|\Omega|)$ and free-stream $(U)$ velocities, namely $\alpha=|\Omega| D /(2 U)$, where $D$ is the diameter of the cylinder. Asymmetric vortex shedding occurs in the wake for $0<\alpha \lesssim 2$; and many experimental and numerical works have shown this alternating vortex shedding over a wide range of Reynolds number (e.g. Coutanceau \& Ménard 1985; Badr et al. 1990; Kang, Choi \& Lee 1999; He et al. 2000; Stojković, Breuer \& Durst 2002; Mittal \& Kumar 2003; Rao et al. 2013). Over a small range of $\alpha \geqslant 4$ there is a secondary region of wake unsteadiness, comprising low-frequency one-sided vortices, as shown by Mittal \& Kumar (2003), Stojković et al. (2003) and Pralits, Brandt \& Giannetti (2010). Numerical simulations have shown that the critical Reynolds number of the secondary instability associated with the spanwise undulation of the von Kármán vortices increases with Reynolds number (El Akoury et al. 2008; Rao et al. 2015). Depending on $\alpha$ and $R e$, there exist a number of steady and unsteady wake modes (Pralits, Giannetti \& Brandt 2013; Rao et al. 2013, 2015).

Bourguet \& Lo Jacono (2014, hereafter BL14) appear to have been the first to conduct a low-Re numerical study of an elastically mounted circular cylinder undergoing forced rotation. This study was undertaken at $R e=100$ over a parameter space spanning the reduced velocity range $4 \leqslant U^{*} \leqslant 34$ and the rotation rate range $0 \leqslant \alpha \leqslant 4$. Here, the reduced velocity is defined b y $U^{*}=U /\left(f_{n w} D\right)$, w ith $U$ the free-stream velocity, $f_{n w}$ the natural frequency of the system in quiescent water and $D$ the cylinder diameter. They found that the time-averaged displacement of the cylinder tended to increase with $\alpha$, as did the time-averaged transverse lift-force coefficient. Non-negligible oscillations (i.e. $A^{*} \equiv A / D \geqslant 0.05$, with $A$ the oscillation amplitude) occurred in both the unstable $(\alpha<1.8)$ and stable $(1.8 \leqslant \alpha \leqslant 3.75)$ flow regimes, based on whether the wake of a non-oscillating rotating cylinder had an unsteady or steady wake. For $\alpha \leqslant 3.75$, the peak amplitude response was found to increase with $\alpha$, and the largest peak amplitude was observed to be $1.9 D$ at $\left(\alpha, U^{*}\right)=(3.75,13)$, three times the peak response of the non-rotating case. Also, they found that large oscillations of the rotating cases were associated with wake-body synchronisations, similar to the lock-in of a non-rotating cylinder. In addition, BL14 examined the wake patterns associated with the effects of cylinder rotation. They observed wake patterns (i.e. $2 \mathrm{~S}, \mathrm{P}+\mathrm{S}$ ) that have commonly been reported in previous VIV studies of a non-rotating cylinder (e.g. Williamson \& Roshko 1988; Blackburn \& Henderson 1999; Mittal \& Kumar 2003; Jauvtis \& Williamson 2004; Dahl et al. 2007). However, they also identified a $\mathrm{n}$ ovel $\mathrm{T}+\mathrm{S} w$ ake $\mathrm{p}$ attern $\mathrm{c}$ omposed of a $\mathrm{t}$ riplet of $\mathrm{v}$ ortices and a single vortex shed per oscillation cycle, which was attributable to the largest amplitude response.

Zhao, Cheng \& Lu (2014c) numerically studied both one- and two-degree-offreedom (1- and 2-DOF) systems with imposed cylinder rotation, again at low Reynolds numbers. At a constant $\alpha$, they observed hysteretic regions in the amplitude response. These regions were similar to those of a non-rotating circular cylinder undergoing VIV when monotonically increasing and then decreasing $U^{*}$. The 2-DOF studies showed significant $\mathrm{d}$ ifferences i $\mathrm{n} \mathrm{v}$ ibration $\mathrm{r}$ esponse $\mathrm{b}$ etween a non-rotating cylinder and cylinders rotating at $\alpha=0.5$ and 1.0.

More recently, Seyed-Aghazadeh \& Modarres-Sadeghi (2015, hereafter SM15) experimentally studied VIV of a rotating cylinder at relatively low Reynolds numbers 


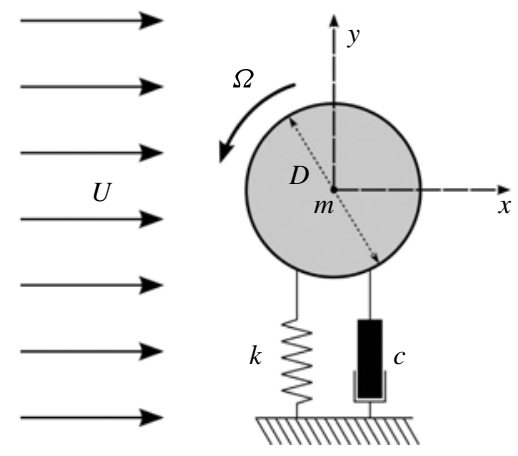

FIGURE 1. Definition sketch for the transverse FIV of a rotating cylinder. The hydroelastic system is simplified as a 1-DOF system constrained to move in the cross-flow direction. The axis of rotation is transverse to both the flow direction ( $x$-axis) and the oscillation axis ( $y$-axis). Here, $U$ is the free-stream velocity, $k$ the spring constant, $D$ the cylinder diameter, $m$ the oscillating mass, $c$ the structural damping and $\Omega$ the angular velocity.

$(R e=350-1000)$. Results were presented showing the vibration response and corresponding flow visualisations in the wake. Of interest, the amplitude response was found to increase only marginally with rotation rate over that of a non-rotating cylinder undergoing VIV, from $A^{*} \simeq 0.5$ to $A^{*} \simeq 0.6$ at $\alpha \simeq 2.4$, and dropping away after this. This result, showing only a minimal effect of rotation, is distinctly different from the previous numerical studies of BL14 and Zhao et al. (2014c).

In summary, previous parameter space studies have investigated the vibration responses and wake modes for both the 1-DOF and 2-DOF oscillation cases, but only at relatively low Reynolds number. Some of these studies appear to be at least superficially contradictory, in terms of amplitude response with rotation rate, peak response and the reduced velocity ranges for significant vibration response. The present work is an attempt to study this problem over a higher-Reynolds-number range, where the VIV response is likely to be less sensitive to Reynolds-number variations. Thus, the findings are likely to be more applicable to typical physical and/or industrial applications, where Reynolds numbers are generally higher. This investigation also aims to study the vibration response and wake structure over a wider range of parameter space $\left(U^{*}, R e\right.$ and $\left.\alpha\right)$ than previous experimental work.

The remainder of the paper is structured as follows. The experimental methodology, including the fluid-structure system modelling, experimental details and a validation study, is presented in $\S 2$. The results are presented in $\S 3$, followed by discussion in $\S 3.4$. Finally, conclusions are drawn in $\S 4$.

\section{Experimental methodology}

\subsection{Fluid-structure system modelling}

A schematic of the fluid-structure system is presented in figure 1. The elastically mounted cylinder is free to oscillate in only one direction transverse to the oncoming free stream. The governing equation of motion can be written as

$$
m \ddot{y}+c \dot{y}+k y=F_{y},
$$

where $F_{y}$ represents the fluid force in the transverse direction, $m$ is the total oscillating mass of the system, $c$ is the structural damping of the system, $k$ is the spring constant and $y$ is the displacement in the transverse direction. 
Side view

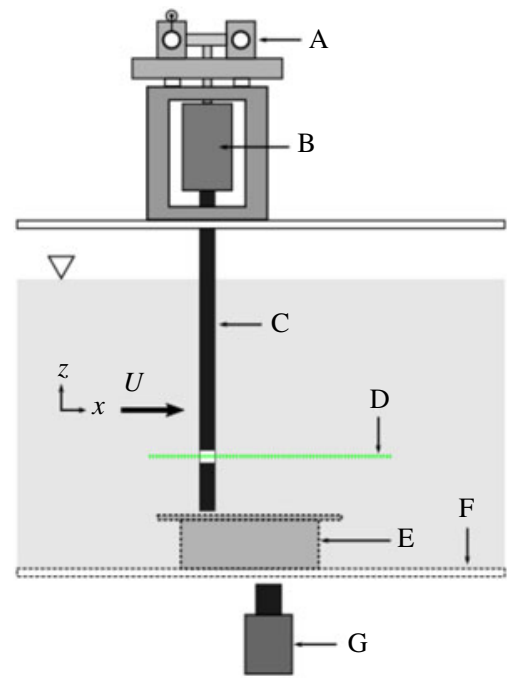

Top view

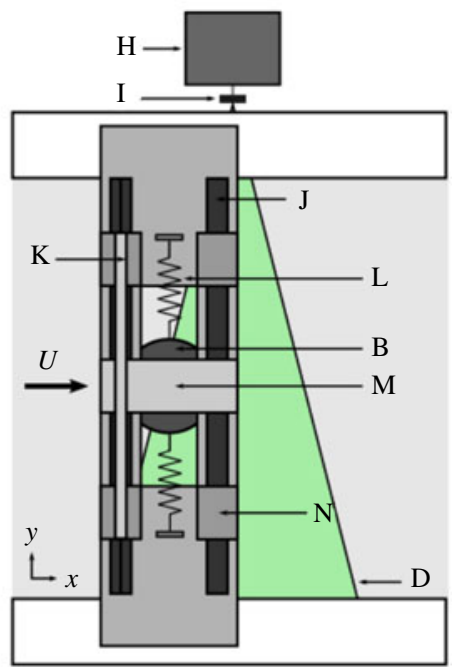

FIgURE 2. (Colour online) Schematic of the experiment (not scaled): A, low-friction air-bearing system; B, cylinder rotation device with built-in motor and optical rotary encoder; C, $30 \mathrm{~mm}$ test cylinder with transparent laser window; D, $532 \mathrm{~nm}$ laser sheet; E, transparent end plate; F, transparent test section window; G, camera; H, $532 \mathrm{~nm}$ laser; I, cylindrical lens; J, carbon shaft; K, linear voltage differential transformer (LVDT); L, stainless-steel springs; $\mathrm{M}$, support carriage; N, air-bearing bushing.

In the present experiments, the hydroelastic system was modelled based on a low-friction air bearing system in conjunction with the recirculating free-surface water channel of the Fluids Laboratory for Aeronautical and Industrial Research (FLAIR), Monash University. The test section of the water channel has dimensions of $600 \mathrm{~mm}$ in width, $800 \mathrm{~mm}$ in depth and $4000 \mathrm{~mm}$ in length. The free-stream velocity in the present experiments could be varied continuously over the range $0.05 \leqslant U \leqslant 0.45 \mathrm{~m} \mathrm{~s}^{-1}$. The free-stream turbulence level was less than $1 \%$. Figure 2 shows a schematic of the experimental set-up, while figure 3 shows an actual photograph of the experimental rig to clarify details. The air-bearing system positioned above the water channel provided low structural damping and constrained the body motion to the cross-flow (transverse) direction. Structural stiffness of the oscillating system was controlled by stainless-steel extension springs attached to both sides of the support carriage and the base of the air-bearing system. More details of this hydroelastic system can be found in Nemes et al. (2012) and Zhao et al. (2014a,b).

The cylinder model used was precision-machined from aluminium tubing and had a diameter of $D=30 \pm 0.01 \mathrm{~mm}$. The immersed length of the cylinder was $L=$ $614 \mathrm{~mm}$, giving an aspect ratio of $L / D \simeq 20.5$. To reduce end effects of the cylinder and to promote parallel vortex shedding, a conditioning platform was placed under the cylinder, and the gap between the cylinder end and the platform surface was set to approximately $1 \mathrm{~mm}(0.03 D$ ) (see Khalak \& Williamson 1997, 1999; Zhao et al. $2014 a, b)$.

The cylinder rotation rate is defined as the ratio of the cylinder surface speed to the free-stream velocity, namely $\alpha=|\Omega| D /(2 U)$. The rotation speed of the cylinder was controlled using a motion control system manufactured by Parker Hannifin (USA) 


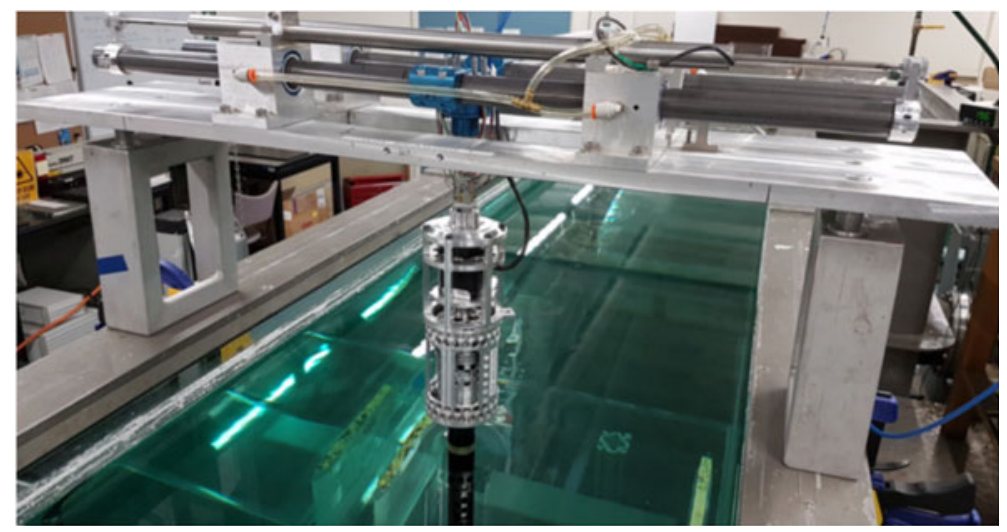

FIGURE 3. (Colour online) A photograph showing the experimental rig used in the present study.

consisting of a controller (model 6K2), a motor drive (model E-DC) and a miniature low-voltage micro-stepping motor (model LV172) with a resolution of 25000 steps per revolution.

The total oscillating mass, including the cylinder model, motor assembly and moving components of the air-bearing system, was $m=2503.6 \mathrm{~g}$ and the displaced mass of the fluid was $m_{d}=433.1 \mathrm{~g}$, resulting in a mass ratio of $m^{*}=m / m_{d}=5.78$. The natural frequencies of the system and the structural damping in both air and water were determined by conducting free-decay tests individually in air and in quiescent water. The natural frequency in quiescent water was found to be $f_{n w}=0.671 \mathrm{~Hz}$. The structural damping ratio in air was $\zeta_{\text {air }} \simeq c /(2 \sqrt{\mathrm{km}})=4.1 \times 10^{-3}$.

The cylinder flexed slightly under large body oscillations, which resulted in a minor deviation between the cross-sectional centroid position of the cylinder at the top, where there was minimal cylinder flexing, and the centroid position of the cylinder at the bottom, where transverse deflection due to flexing was largest. This was quantified by measuring the difference in the centroid positions at the top and bottom using a linear variable differential transformer (LVDT) and a high-speed camera (see $\S 2.2$ for further details), respectively. In the worst case $\left(U^{*}=6.0\right)$ the deviation was measured as $\sim 0.1 D$, while the peak-to-peak oscillation measured $2.8 D$, i.e. approximately $3.5 \%$. Alternatively, this represented a deflection of the cylinder over its length of less than $0.3^{\circ}$.

\subsection{Data acquisition and processing methods}

The data acquisition and the control of the free-stream velocity and motor rotation speed over the $U^{*}-\alpha$ parameter space were automated using customised LabVIEW (National Instruments, USA) programs. The reduced velocity was varied over the range $2.5 \leqslant U^{*} \leqslant 14$ in increments between 0.25 and 1 (depending on structural response regimes). The corresponding Reynolds-number range was $1132 \leqslant R e \leqslant 6313$. Note that the Reynolds number is defined by $R e=U D / v$, where $v$ is the kinematic viscosity of the fluid. Fluctuations in the kinematic viscosity with temperature were considered minimal, with temperature fluctuations of less than $\pm 0.5^{\circ} \mathrm{C}$ while the experiments were undertaken. The cylinder rotation rate was varied over the range $0 \leqslant \alpha \leqslant 4.2$ in increments of 0.05 . 
The body displacement was measured using a non-contact (magnetostrictive) LVDT. The accuracy of the LVDT was within $\pm 0.01 \%$ of the $250 \mathrm{~mm}$ range available (see Zhao et al. 2014a,b). The rotation speed was measured using a digital optical rotary encoder (model E5-1000, US Digital, USA) with a resolution of 4000 counts per revolution. The sampling frequency for all measurements was set to $100 \mathrm{~Hz}$. For each point in the $U^{*}-\alpha$ parameter space, the structural response was measured for at least $300 \mathrm{~s}$, which amounted to more than 300 oscillation cycles. Sufficient transition time (180 s) was allowed between changing either $\alpha$ or $U^{*}$ to enable the VIV response to fully develop before recording the signal sequences. To remove high-frequency electrical noise, a low-pass Butterworth filter of o rder $4 \mathrm{w}$ ith a $\mathrm{c}$ ut-off f requency of $2 \mathrm{~Hz}$ was applied to all analogue signals prior to further processing. Fast Fourier transforms (FFTs) were used to extract body oscillation frequencies from the LVDT signals.

The particle image velocimetry (PIV) technique was employed to capture flow structures in the near wake of the cylinder. The flow w as s eeded $w$ ith hollow microspheres (Sphericel 110P8, Potters Industries Inc.) having a normal diameter of $13 \mu \mathrm{m}$ and a specific w eight of $1.1 \mathrm{~g} \mathrm{~m}^{-3}$. T he $\mathrm{p}$ articles w ere i lluminated b y a $2 \mathrm{~mm}$ thick horizontal planar laser sheet from two miniature $\mathrm{Ng}$ :YAG pulse lasers (Continuum Minilite II, USA), while imaging was performed using a high-speed camera (Dimax S4, PCO AG, Germany) with a resolution of 2016 pixel $\times 2016$ pixel. The camera was equipped with a $50 \mathrm{~mm}$ lens (Nikon Corporation, Japan), giving a magnification of $9.71 \mathrm{p}$ ixel $\mathrm{m} \mathrm{m}^{-1} \mathrm{f}$ or $\mathrm{t}$ he fi eld of vi ew of in terest. Th e PI V image pairs were captured at $14 \mathrm{~Hz}$ using a TTL pulse generator. For each PIV imaging location in the $U^{*}-\alpha$ space, 3140 image pairs were recorded for quantitative analysis. The imaging data were processed using validated in-house PIV software developed by Fouras, Lo Jacono \& Hourigan (2008). More details of the method used for identifying the wake structures are further provided in $\S 3.2$.

\subsection{Validation}

The vibration response of a non-rotating circular cylinder undergoing VIV is compared against previous work by Khalak \& Williamson (1997, 1999) and Zhao et al. (2014b) in figure $4 . \mathrm{F}$ igure $4(a) \mathrm{p}$ resents t he a mplitude $\mathrm{r}$ esponse a s a $\mathrm{f}$ unction o f reduced velocity and shows the current experimental configuration p roduces $n$ on-rotating VIV results in good agreement with previous VIV studies. Here, it is important to note the effect of the mass ratio, which is necessarily higher here than for some of the comparison studies because of the added weight arising from the motor assembly. At the lowest $m^{*}$ achievable, of $m^{*}=5.78$, the typical VIV amplitude response branches of a non-rotating cylinder are seen. The initial branch exists for $U^{*}<4$.8. At $m^{*}=$ 5.78 , the upper branch covers the range $U^{*}=4.8-6.4$; the lower response branch lies within the range $6.4 \leqslant U^{*} \leqslant 9.5$; and the desynchronised region occurs for $U^{*}>9.5$. Previous studies by Khalak \& Williamson (1997) and Zhao et al. (2014b) at $m^{*}=2.4$ showed a peak amplitude response of $A^{*} \approx 0.95$. The mean of the highest $10 \%$ of peak amplitude response $\left(A_{10}^{*}\right)$ of the current system is $A_{10}^{*}=0.82$. This agrees with previous studies on the effects of mass and damping. As the mass and damping ratios are increased, the magnitude of the amplitude response decreases and the range of $U^{*}$ over which the system self-excites reduces (Feng 1968). A hysteretic transition between the initial and upper branch occurs at $U^{*} \approx 4.8$ and is accompanied by a jump in $A_{10}^{*}$ from 0.25 to 0.79 . The transition from the upper to lower branch causes a reduction in $A_{10}^{*}$ from 0.78 to 0.55 , and the desynchronisation of the system at 

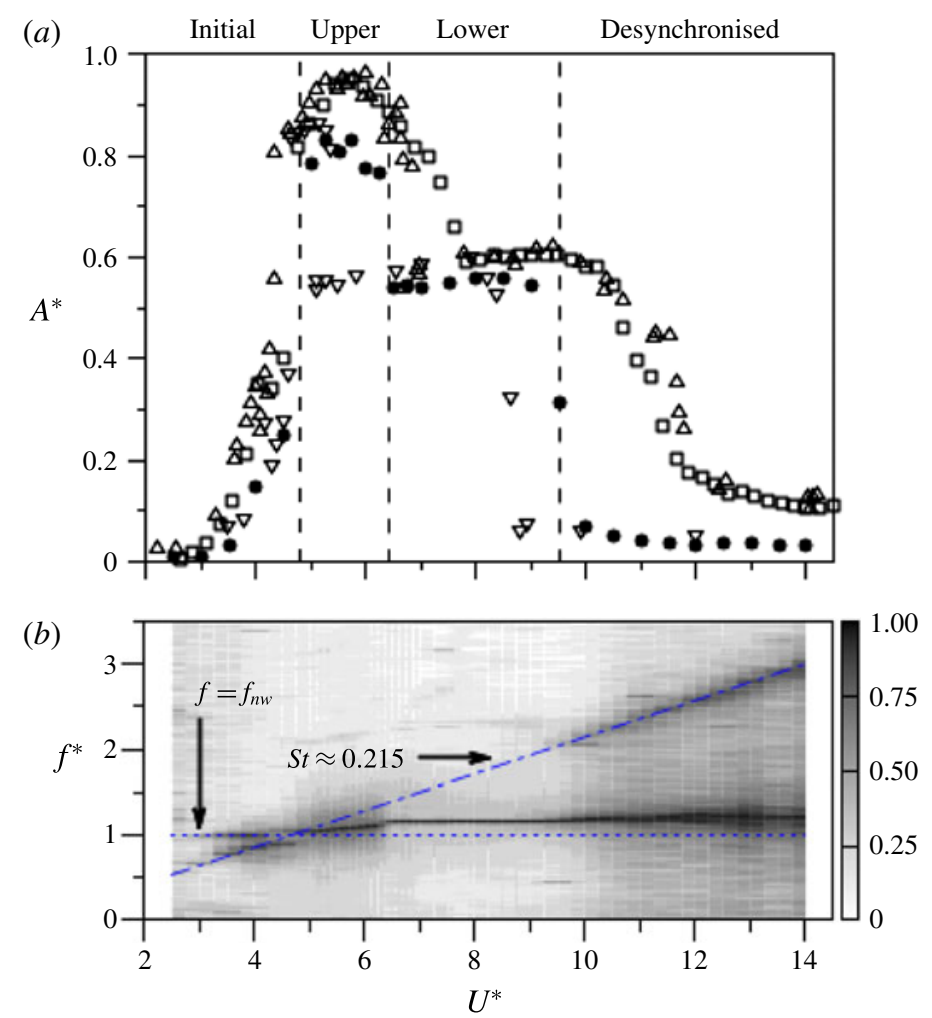

FIGURE 4. (Colour online) Vibration response of a non-rotating circular cylinder undergoing VIV. (a) The normalised amplitude response $\left(A^{*}\right)$ as a function of reduced velocity $\left(U^{*}\right)$ : $\bullet$, the mean of the highest $10 \%$ of oscillation amplitude in the present study with $m^{*}=5.78$ and $\zeta=0.0041 ; \square$, results from Zhao et al. (2014b) with $m^{*}=$ 2.4 and $\zeta=0.00243 ; \Delta$, results from Khalak \& Williamson (1997) with $m^{*}=2.4$ and $\zeta=0.0045 ; \nabla$, results from Khalak \& Williamson (1999) with $m^{*}=10.3$ and $\zeta=0.00165$. The vertical dashed lines represent the boundaries of branches for the present study. (b) A PSD contour map of $A^{*}$ as a function of $f^{*}$ and $U^{*}$. Essentially normalised power spectra are assembled together horizontally to construct this map. Note that the PSD is normalised by the peak value at each $U^{*}$.

$U^{*} \approx 9.5$ is accompanied by a drop in $A_{10}^{*}$ from 0.54 to $\sim 0.07$. This comparison with results from previous literature shows that the current air-bearing system reproduces an amplitude response consistent with previous careful studies.

Figure 4(b) presents a power spectral density (PSD) contour plot of the normalised cylinder displacement as a function of the normalised frequency response $f^{*}$ and reduced velocity $U^{*}$. This consists of individual vertically aligned greyscale-coded power spectra for each $U^{*}$ stacked together horizontally to show the frequency response of the system, with darker regions representing frequencies of higher power. The narrowness or broadness of the spectral peaks is also clear from the colour gradation. The variation of the shedding frequency ratio $\left(f_{s h} / f\right)$ with $U^{*}$ is plotted as a dot-dashed line in figure $4(b)$ to highlight $U^{*}$ regions where $f \approx f_{s h}$. To highlight $U^{*}$ ranges where $f$ locks to $f_{n w}$ (i.e. $f \approx f_{n w}$ ), the $f^{*}=1$ curve is shown as a dotted line in figure $4(b)$. The body's frequency response $(f)$ follows the shedding frequency $\left(f \approx f_{\text {sh }}\right)$ until the end of the initial amplitude response branch, where it begins to bend 
towards $f_{n w}$ and subsequently lock in to it $\left(f \approx f_{n w}\right)$. The frequency response remains synchronised throughout the upper and lower amplitude response branches until the desynchronised region is reached. At that point, the body frequency response deviates from $f_{n w}$, returning to follow $f_{s h}$, although there is still a broad spectral peak close to the natural shedding frequency. For the current experiment, the mean Strouhal number, defined b y $S t=f_{s h} D / U, \mathrm{w}$ as $\mathrm{m}$ easured a s $S t \approx 0.215 \mathrm{f}$ or $\mathrm{t}$ he $R e \mathrm{r}$ ange investigated; this agrees with the known $S t$ value in the same $R e$ range.

\section{Results}

The vibration response and time-averaged position of the cylinder are presented in $\S 3.1$, and the wake modes and structure are explored in $\S 3.2$. A specific section is devoted to the wake state in the upper branch where intermittent behaviour is observed to occur $(\$ 3.3)$, prior to a short discussion comparing current results with those from previous related studies in the following section $(\$ 3.4)$.

\subsection{Vibration response}

The time-averaged displacement of the cylinder from its non-rotating neutral position $(\bar{y})$ is discussed first, followed by the observed vibration response, i.e. the oscillation amplitude and frequency response, as $U^{*}$ and $\alpha$ are varied. The oscillations observed in the current study are typically broadly periodic although there is some variation of the waveform from cycle to cycle. When a cylindrical body is rotated about its axis in a fluid flow, an asymmetric pressure distribution is generated. This uneven pressure distribution is the result of fluid being accelerated by the body rotation on the leeward side, and decelerated with flow separation from the body on the windward side. This is generally known as the Magnus effect, and it has been extensively studied over the past century (e.g. Tietjens \& Prandtl 1957; Coutanceau \& Ménard 1985; Badr et al. 1990; Kang et al. 1999; He et al. 2000; Stojković et al. 2002; Mittal \& Kumar 2003; Rao et al. 2013). A net transverse force is generated by the asymmetric pressure distribution. The magnitude of the force increases with $\alpha$; therefore, it is expected that, at any $U^{*}$, an increase in $\alpha$ will result in an increase in the magnitude of $\bar{y}$. Figure 5 presents $\bar{y}$ as a function of $U^{*}$ at varying $\alpha$. This shows that $\bar{y}$ increases in magnitude with $\alpha$ as expected due to the increase in the mean cross-flow force. This general behaviour agrees with rotating VIV results from low-Reynolds-number $(\operatorname{Re}=100)$ simulations of BL14, although the current experimental offsets are generally larger than the numerical ones. In figure 5, the $R e=100$ numerical predictions for $\alpha=2.0$ (dashed) and $\alpha=4.0$ (solid) are overlaid for comparison. The measured experimental variation of SM15 for $\alpha=2.0$ is also overlaid (dot-dashed line). This is closer to the $R e=100$ numerical result than the present variation, perhaps consistent with the lower-Reynolds-number range $(R e=350-1000)$ of that study.

The oscillatory component of the motion of the cylinder is characterised in figures 6 and 7. Figure 6 shows how the vibration response varies as a function of $U^{*}$. The means of the highest $10 \%$ of normalised amplitude response peaks $\left(A_{10}^{*}\right)$ about their time-averaged positions $(\bar{y})$ are presented as a function of $U^{*}$ and $\alpha$ in figure $6(a)$. The amplitude response for a number of different cases $(\alpha=0,1.0,2.0$ and 3.0) are highlighted by dashed lines to help clarify the overall behaviour with rotation rate. Large amplitude oscillations are observed over a broad range of $U^{*}$ and $\alpha$. The peak amplitude first increases as the rotation rate is increased from $\alpha=0$, while beyond $\alpha=$ 2 there is a decrease in the peak response. Overall, the amplitude response is similar to the non-rotating VIV case for $\alpha \leqslant 2.0$. While distorted by the effects of rotation, 


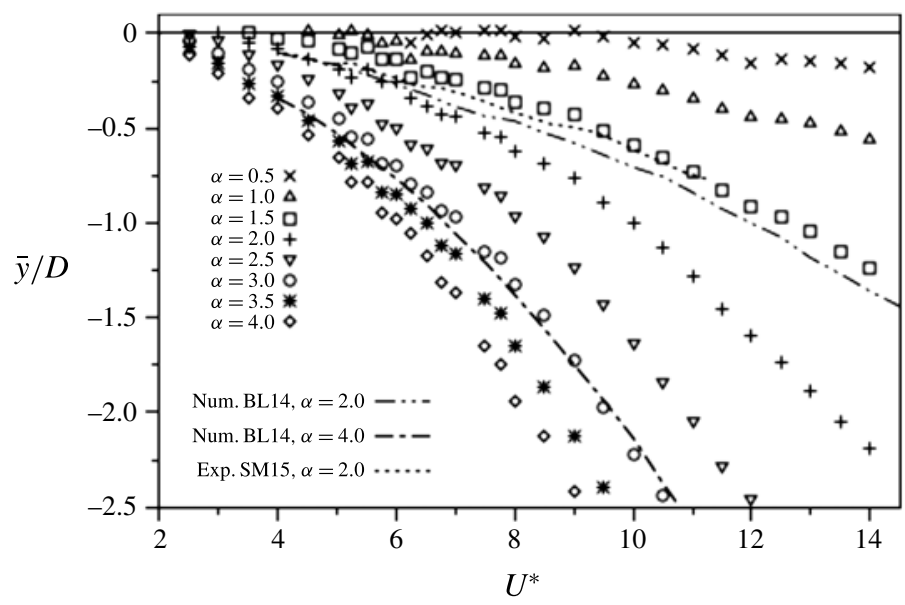

FIGURE 5. The time-averaged displacement of the cylinder $(\bar{y})$ as a function of reduced velocity $\left(U^{*}\right)$ at different rotation rates $(\alpha)$. Numerical predictions BL14 for $R e=100$ at $\alpha=2.0$ and 4.0 are shown by the dot-dashed lines. The dotted line shows experimental results of SM15 at $R e=350-1000, \alpha=2.0$ for comparison.
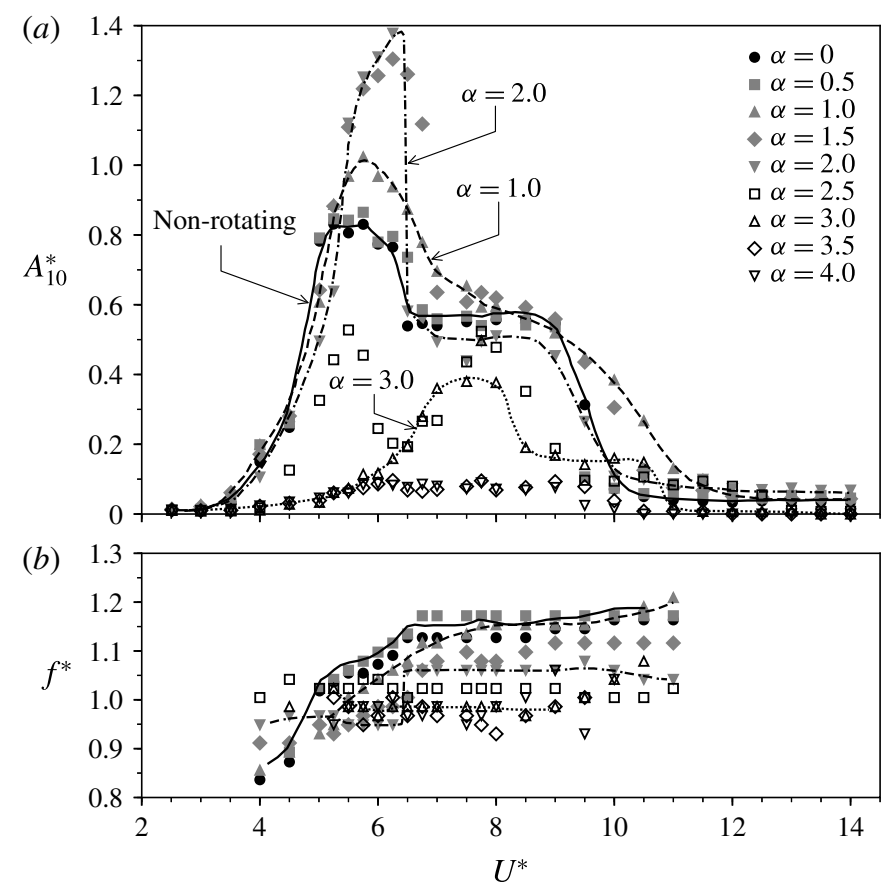

FIGURE 6. The vibration response of an elastically mounted circular cylinder undergoing constant rotation as a function of reduced velocity $\left(U^{*}\right)$ at different rotation rates $(\alpha)$. (a) The normalised amplitude response $\left(A_{10}^{*}\right)$. (b) The normalised frequency response $\left(f^{*}\right)$. Approximate fits to cases $\alpha=0,1.0,2.0$ and 3.0 are shown by the labelled dashed lines.

the three amplitude response branches, i.e. the initial, upper and lower branches, can be clearly identified. Over the rotation rate range $0<\alpha \leqslant 1.5$, an increase in $\alpha$ results in an increase in peak amplitude response, transitions between the upper and lower 
branch and the desynchronisation become less distinct, and the width of the $U^{*}$ range over which excitation occurs increases. For rotation rates between $1.5<\alpha \leqslant 2.0$, the peak amplitude increases to higher values than seen at lower $\alpha$. The transition between upper and lower branches is abrupt, but the range of $U^{*}$ at which excitation occurs is similar to that of a non-rotating cylinder. Up to a rotation rate of $\alpha \approx 2.0$, the $U^{*}$ range corresponding to the amplitude peak increases with $\alpha$. In the range $2<U^{*} \leqslant 3$, the amplitude response decreases significantly, a nd the $\mathrm{r}$ esponse $\mathrm{c}$ urve $\mathrm{s}$ hape $\mathrm{n}$ o longer resembles that seen for non-rotating VIV. Instead of the typical two- or three-branch response, in this range of $\alpha$ these are replaced with a twin-peak $(\alpha=2.5)$ and a small single-peak $(\alpha=3.0)$ response. Also, the range of reduced velocity at which excitation occurs becomes narrower. For $\alpha>3.0$, body excitation is minimal.

The normalised frequency response about its time-averaged position is presented as a function of $U^{*}$ and $\alpha$ in figure $6(b)$. A $\mathrm{s}$ f or t he a mplitude $\mathrm{r}$ esponse curves, the frequency variations for the non-rotating case and $\alpha=1.0,2.0$ and 3.0 are highlighted by dashed lines. For the non-rotating case, again there is good agreement with previous VIV studies. The frequency response follows the non-rotating VIV response and the response globally decreases with increasing $\alpha$. This is also in good agreement with the numerical results found by BL14 and experiments of SM15.

A study of the vibration response at fixed $\alpha$ i ncrements $\mathrm{c}$ an $\mathrm{b}$ etter s how the effects of rotation on the vibration response and how each $\alpha$ increment compares to the nonrotating VIV case over the tested $U^{*}$ range. The normalised vibration response about its time-averaged position at several $U^{*}$ of interest were selected and are presented as a function of $\alpha$ and $U^{*}$ in figure 7 . T he s election o $\mathrm{ft}$ hese $\mathrm{v}$ alues of $U^{*}$ was based on how each represents the vibration response at similar $U^{*}$, and how well these cases show the response progression with increasing $U^{*}$. Figure 7(ai) shows $A_{10}^{*}$ as a function of $\alpha$ at $U^{*}=4.00$. At this reduced velocity, the amplitude response is small $\left(A^{*} \lesssim 0.2\right)$ with increased rotation further suppressing oscillations beyond $\alpha=2.3$. Figure 7(aii) shows $f^{*}$ as a function of $\alpha$ at $U^{*}=4.00$. When the rotation rate is increased, the body frequency response converges towards $f_{n w}$ with an associated decreased amplitude response. This trend was observed in the initial branch and at the beginning of the upper branch.

Figure $7(b \mathrm{i}, \mathrm{ii})$ show that the amplitude increases significantly u p to a r otation rate of $\alpha \approx 2.0$ and abruptly decreases thereafter (highlighted by the vertical dashed line in figure $7 \mathrm{bi}$ ). The highest a mplitude observed in the c urrent s tudy occurred at $U^{*}=6.25$. Specifically, $\mathrm{t}$ he a mplitude i ncreased $\mathrm{f} \operatorname{rom} A_{10}^{*}=0.79 \mathrm{i} \mathrm{n} \mathrm{t} \mathrm{he} \mathrm{n}$ on-rotating c ase $\mathrm{t}$ o a peak value of $A_{10}^{*}=1.39$ at $\alpha=2.0$. With further increase in $\alpha$, the amplitude drops to $A_{10}^{*} \approx 0.3$ at $\alpha \approx 2.3$.

The frequency response decreases with increasing $\alpha$ towards $f_{n w}$. There is a small jump in the frequency response accompanying the drop in amplitude response (highlighted by the vertical dashed line in figure $7 \mathrm{bii}$ ). A s t he f requency initially converges towards the natural frequency of the structure, the oscillation locks in to the natural frequency, resulting in an increase in amplitude response. As the rotation rate is increased past $\alpha \approx 2.25$, the wake mode changes (refer to $\$ 3.2$ ) and the response frequency is no longer locked in with the natural frequency of the structure.

When the reduced velocity is further increased to the onset of the lower amplitude response branch $\left(U^{*}=6.5\right)$, there is an increase in the amplitude response. Figure $7(c \mathrm{i})$ shows that the amplitude response does drop following the peak, but in this case the sharp drop occurs at a lower rotation rate $(\alpha \approx 1.7)$ and is followed by a plateau between the peak and the lower amplitude regions. Over the range of $\alpha$ where the amplitude plateau exists, the frequency response locks in with the natural frequency 


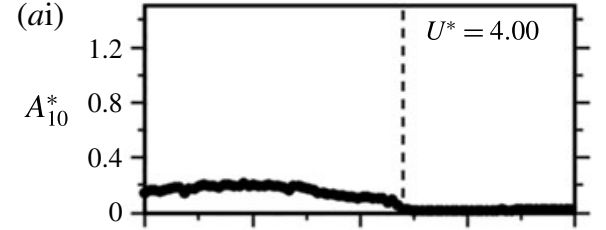

(aii)
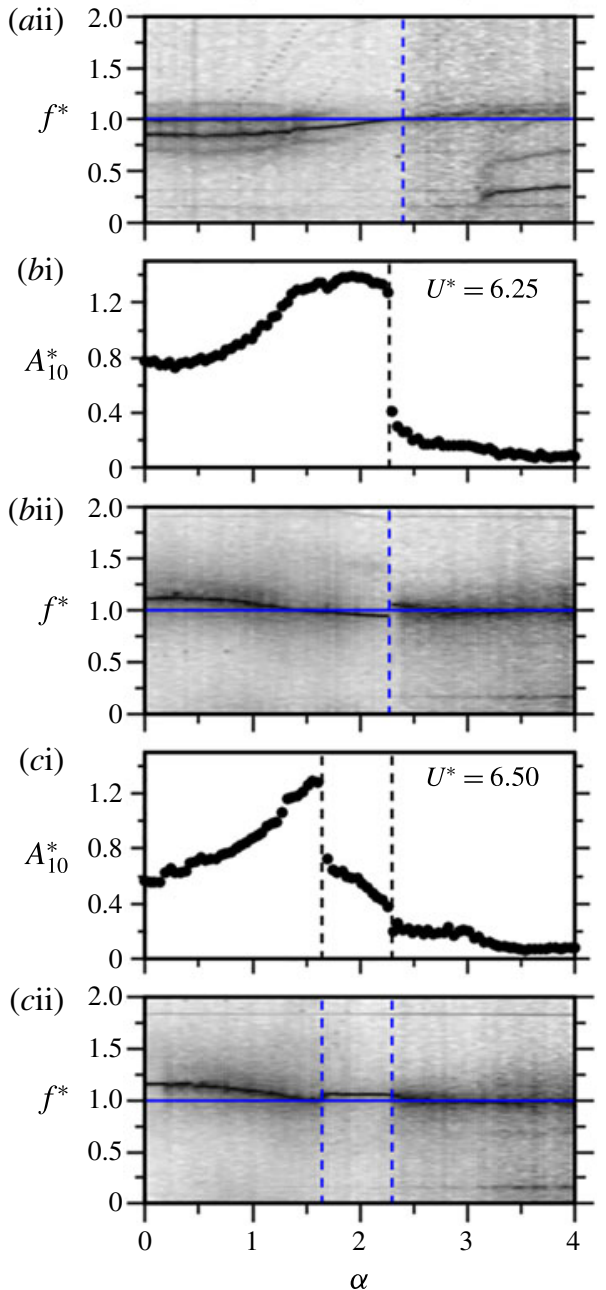

(di)

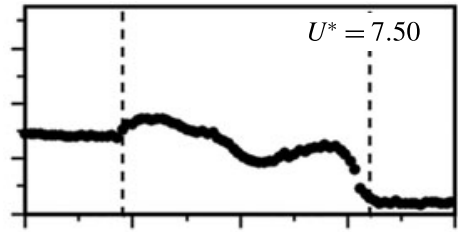

(dii)

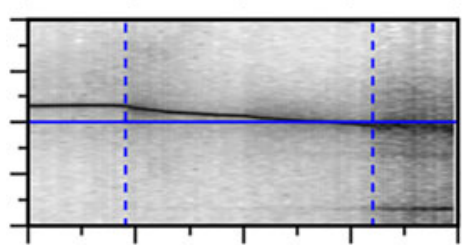

(ei)

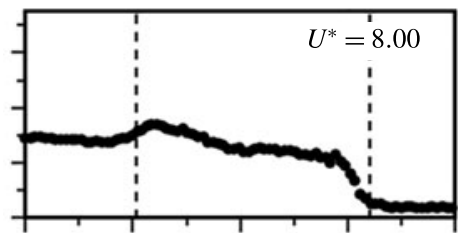

(eii)

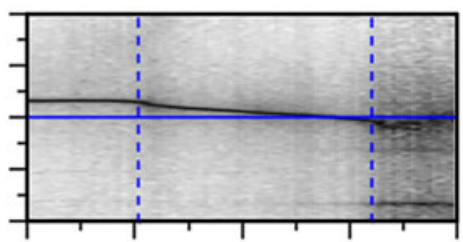

(fi)

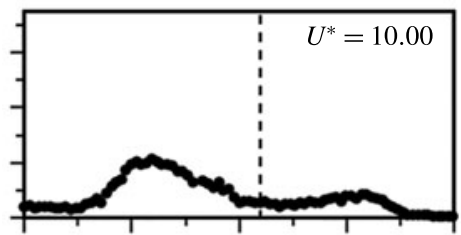

(fii)

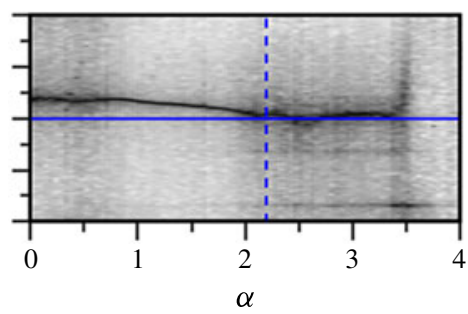

FIGURE 7. (Colour online) The vibration response of an elastically mounted circular cylinder undergoing constant rotation as a function of rotation rate $(\alpha)$ at selected reduced velocities $\left(U^{*}\right)$. In each reduced velocity case, panel (i) is the normalised amplitude response $\left(A_{10}^{*}\right)$ and panel (ii) is the PSD contour of the normalised reduced velocity $\left(f^{*}\right)$ normalised by the peak power. The horizontal solid lines in panels (ii) indicate $f^{*}=1$ (or $\left.f=f_{n w}\right)$. The vertical dashed lines highlight features of interest discussed in the text.

of the structure, i.e. $f \approx f_{n w}$. When the rotation rate is increased past $\alpha \approx 2.25$ (second vertical dotted line), the frequency response is similar to that of the upper branch. From $\alpha \approx 2.25$ onwards, $f^{*}$ in both cases begins at a value slightly above $f^{*}=1$, then it gradually decreases to below $f^{*}=1$ as $\alpha$ is increased. 
When the reduced velocity is further increased to the $U^{*}=7.50$ (lower branch), the vibration response is different from that seen at the beginning of the lower response branch $\left(U^{*}=6.5\right)$. Figure $7(d \mathrm{i})$ exhibits two amplitude peaks, the second appearing at a higher $\alpha$ than the peaks seen at lower $U^{*}$. The amplitude response is relatively unresponsive to rotation rates up to $\alpha \approx 0.8$ (highlighted by the vertical dashed line in figure $7 d i$ ). T he s econd peak a ppears w hen the rotation $r$ ate is close to $\alpha \approx 2.75$. The frequency response is similar to that of the amplitude, with little effect of rotation up to $\alpha \approx 0.8$. Further increase in $\alpha$ is accompanied by a gradual reduction in $f^{*}$ down to and then below $f^{*}=1\left(f=f_{n w}\right)$. The location of the second amplitude peak and the point at which $f$ decreases past $f_{n w}$ suggests that the appearance of the second amplitude peak is due to $f$ approaching $f_{n w}$. Similar trends were observed at $U^{*}=8.0$; however, the second peak becomes less distinct.

At $U^{*}=10.0$ (desynchronised region), the amplitude and frequency responses tend to be similar to those in the lower branch, as can be observed from figure $7(f \mathrm{i}, \mathrm{ii})$. However, the magnitude of the amplitude is much lower than in the lower branch. Body rotation does not appear to significantly a ffect $t$ he a mplitude $r$ esponse of the cylinder when the reduced velocity lies in the initial branch. However, in the desynchronised region there is an unexpected response. With increasing rotation rate, the amplitude response increases from approximately 0.1 to 0.5 , while the frequency response is not close to the natural frequency of the structure.

To understand how the wake structure affects the vibration response of the cylinder, the wake patterns are examined in the following section.

\subsection{Wake structures}

Vortex shedding structures in the wake are significant a s t hey i nfluence th e body vibration response of an elastically mounted structure. Williamson \& Roshko (1988), Badr et al. (1990), Khalak \& Williamson (1999), Carberry et al. (2001), Mittal \& Kumar (2003), Rao et al. (2013) and Zhao et al. (2014a) have discovered and categorised the wake patterns of forced rotation on rigidly mounted cylinders and non-rotating cylinders undergoing free and forced vibrations. The wake structure can vary significantly f rom t he K ármán $\mathrm{v}$ ortex s treets $\mathrm{t}$ ypically o bserved f or a stationary cylinder. BL14 have shown that an elastically mounted cylinder undergoing forced rotation can exhibit wake structures previously observed in forced vibration, free vibration and forced rotation studies. To better understand how the wake modes interact with the body vibration response, the wake modes have been mapped against the primary independent variables, $U^{*}$ and $\alpha$.

Figure 8 is a contour map of the mean response amplitude in $U^{*}-\alpha$ parameter space. The dashed lines are iso-amplitude contours. The wake patterns observed for selected PIV datasets (discussed in more detail below) are marked on this figure to indicate how the wake state affects the amplitude response. The solid lines represent approximate boundaries of regions with the same wake state. These are mainly drawn to aid in the interpretation of how the wake state and amplitude response are related.

As indicated above, a large number of PIV snapshots were gathered at a high sampling frequency capturing approximately 12-15 snapshots per cycle for typically 200-300 cycles (3140 images gathered). The recording of the PIV snapshots were synchronised with the cylinder motion. 


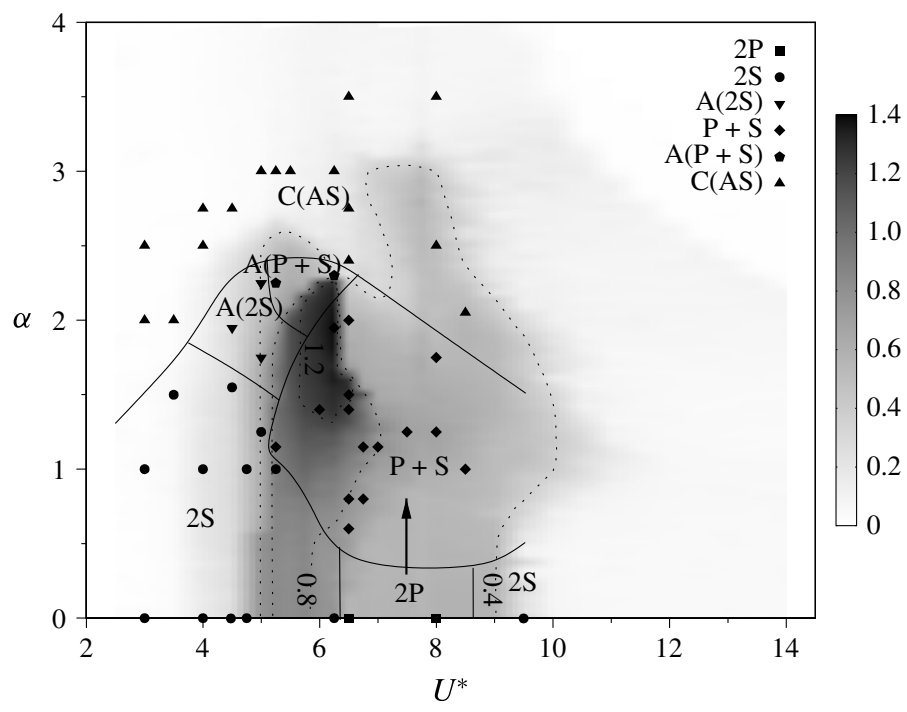

FIGURE 8. The wake patterns observed based on PIV data with approximate boundaries shown by the solid lines. This overlays a greyscale contour map of the mean peak amplitude, in $U^{*}-\alpha$ parameter space, with amplitude levels indicated by the dashed lines. There appears to be a gradual change from $2 \mathrm{P}$ to $\mathrm{P}+\mathrm{S}$ as the rotation is increased, causing a deflection of the wake away from the streamwise centreline.

To investigate the wake state beyond the PIV images, the classical snapshot method (Sirovich 1987; Holmes et al. 2012) was followed using the MODRED 2.0.1 library to decompose the fields. The velocity field, $\boldsymbol{u}(\boldsymbol{x}, t)$, is decomposed as follows:

$$
\boldsymbol{u}(\boldsymbol{x}, t)=\sum_{i=0}^{N} \phi_{i}(t) \boldsymbol{\psi}_{i}(\boldsymbol{x}) .
$$

Here $\boldsymbol{x}$ is the illuminating laser plane coordinates, $N=3140$ is the number of snapshots taken, $\boldsymbol{\psi}(\boldsymbol{x})$ are the proper orthogonal decomposition (POD) spatial modes and $\phi(t)$ are the temporal mode coefficients. In the presence of periodic or near-periodic flow, the mean flow is approximately represented by $\boldsymbol{\psi}_{0}(\boldsymbol{x})$, whereas, in terms of the shedding process, the first pair of temporal modes, $\phi_{1}$ and $\phi_{2}$, together represent the most energetic shedding mode. The subsequent pair is often a harmonic of the first pair. Therefore, the first temporal coefficients $\left(\phi_{1}-\phi_{4}\right)$ are often sufficient to assess the flow state and to reorder the velocity/vorticity fields into similar phases, as shown in Legrand et al. (2011), Sherry et al. (2013) and Venning et al. (2015). All the PIV data were subsequently analysed with the POD approach and phase averaging was based on the temporal evolution of the first two modes associated with the main shedding behaviour. In the present study, the images in each set were categorised into 12 phases for averaging.

A non-rotating cylinder undergoing VIV shows hysteresis and wake mode switching in the transition regions between the amplitude response branches (Feng 1968; Khalak \& Williamson 1999; Williamson \& Govardhan 2004). A primary focus of the current study is to determine the wake patterns in $U^{*}-\alpha$ parameter space.

In low amplitude response regions, i.e. at reduced velocities associated with the initial response branch $\left(U^{*}<4.8\right)$ and in the desynchronised region $\left(U^{*} \geqslant 9.5\right)$, the shedding mode typically found for non-rotating VIV was observed up to a rotation 

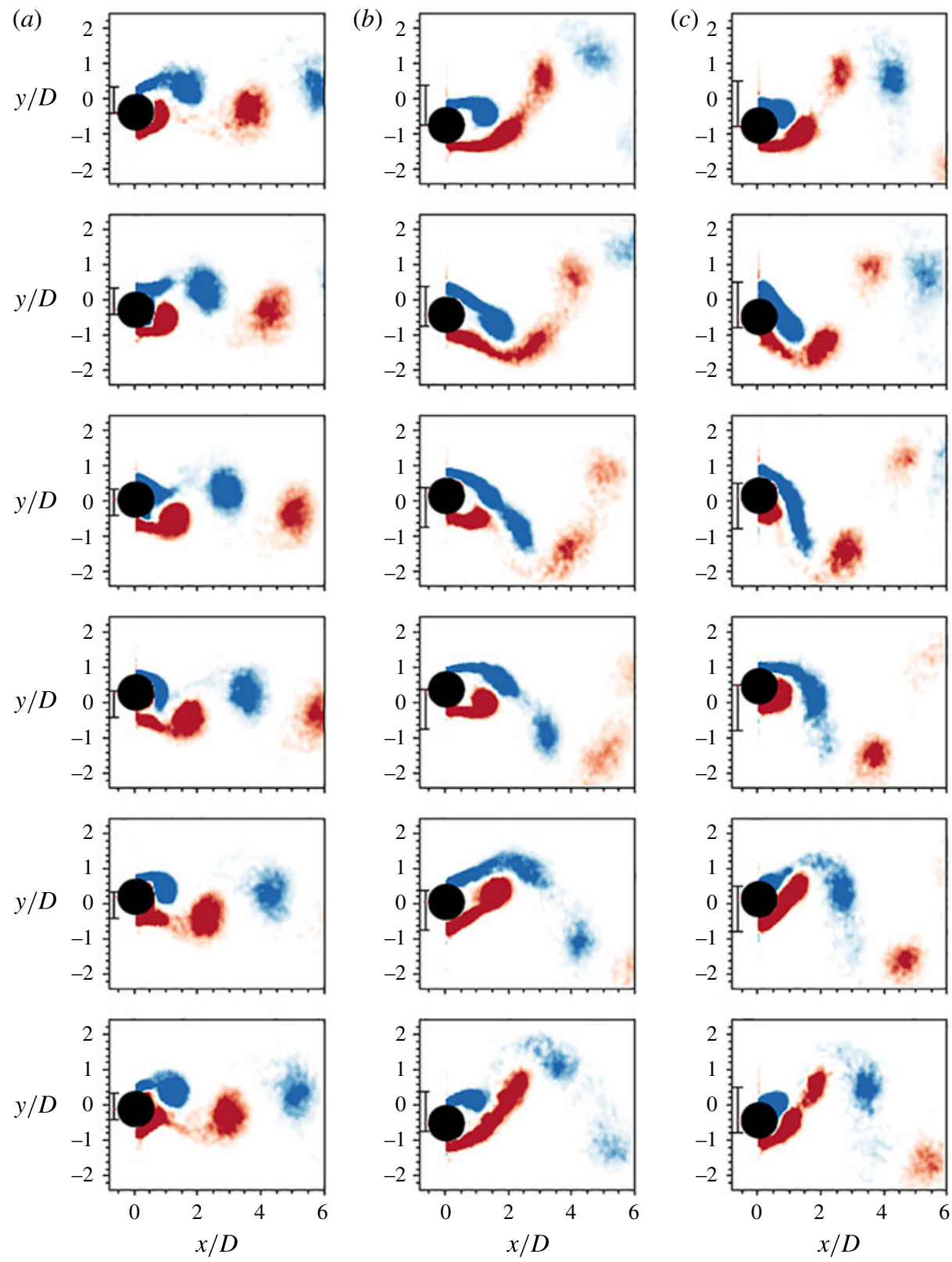

FIgURE 9. (Colour online) Phase-averaged spanwise vorticity contours of a $2 \mathrm{~S}$ mode at $U^{*}=5.0, \quad \alpha=1.0(a), 2 \mathrm{P}$ mode at $U^{*}=8.0, \alpha=0.0(b)$ and $\mathrm{P}+\mathrm{S}$ mode at $U^{*}=6.5, \alpha=0.6(c)$ over an oscillation cycle. Cylinder rotation is anticlockwise. The normalised vorticity range is $\omega^{*}=\omega D / U \in[-1,1]$, where $\omega$ is the vorticity. Near the cylinder the vorticity exceeds these values, so the contour levels are clipped to this range to allow downstream vortices to be visible.

rate of $\alpha \approx 2.0$. This wake is characterised by shedding two single counter-rotating vortices per oscillation cycle (figure 9). This is referred to as the $2 S$ mode. This is the standard shedding mode commonly found in both stationary and non-rotating VIV studies. 

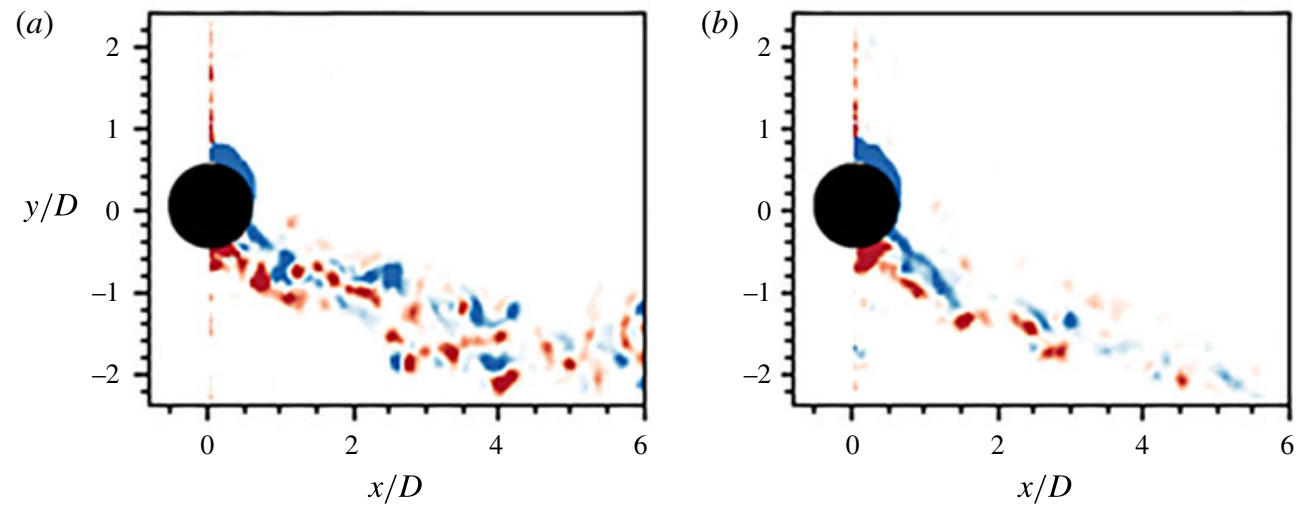

FIgURE 10. (Colour online) Instantaneous isocontours of the $\mathrm{C}(\mathrm{AS})$ mode at $U^{*}=$ $4.00, \alpha=2.75$. The $\mathrm{C}(\mathrm{AS})$ wake pattern is of a chaotic nature and consists of small vortices patched around a curve. The normalised vorticity range is $\omega^{*}=\omega D / U \in[-1,1]$.

When the rotation rate is above $\alpha \approx 2.0$ for the same reduced velocity ranges $\left(U^{*}<\right.$ $4.8, U^{*} \geqslant 9.5$ ), a new wake pattern characterised by shedding of small asymmetric vortices was observed. It appears similar to the shedding mode observed in the second region of wake instability reported by Mittal \& Kumar (2003), although that mode was observed at significantly higher rotation rates $(\alpha \sim 4.5)$. When the flow first undergoes transition to this mode, i.e. at its boundary with other wake modes, the vortices shed are larger and more periodic. As the rotation rate is increased further, the shed vortices become smaller and their shedding is more chaotic. However, it was observed that the wake switches between a wider and a narrower state, with the former associated with the shedding of smaller vortices. The coalescence of these small asymmetric vortices, together with its wake switching, has led to this mode to be named $\mathrm{C}(\mathrm{AS})$. Instantaneous spanwise vorticity contours for this mode are illustrated in figure 10: $(b)$ illustrates the wider wake state; and $(a)$ shows the narrower state.

At moderate reduced velocities associated with the lower amplitude response branch $(6.5 \leqslant \alpha \leqslant 9.5)$, where $0.4<A_{10}^{*} \leqslant 0.6$ and the rotation rate is below $\alpha \leqslant 0.5$, two pairs of counter-rotating vortices are shed per oscillation cycle (figure 9). This resembles the standard 2P mode reported in previous non-rotating VIV studies (e.g. Williamson \& Roshko 1988; Khalak \& Williamson 1999).

At moderate rotation rates $(1.25 \leqslant \alpha<2.25)$ a dominant wake mode persists across the vibration region $\left(5.0<U^{*} \leqslant 9.0\right)$, for which the wake and body motions synchronise. Here, the wake is characterised by the $\mathrm{P}+\mathrm{S}$ mode as shown in figure 9 . This wake mode is composed of a pair $(\mathrm{P})$ of counter-rotating vortices and a single (S) vortex shed per oscillation cycle. Again, this mode has been previously observed in free and forced vibration studies (see Williamson \& Roshko 1988). It has also been observed for the low-Reynolds-number rotating VIV simulations of BL14 and experiments of SM15.

Over the same reduced velocity range $\left(5.0<U^{*} \leqslant 9.0\right)$, the $\mathrm{C}(\mathrm{AS})$ wake mode appears when the rotation rate is increased above $\alpha>2.25$.

From figures 7 and 8 , it is evident that large changes in the oscillation amplitude response correspond to changes in the wake mode. For example, in the case of $U^{*}=$ 6.25 , the drop in amplitude and jump in frequency response near $\alpha=2.25$ coincide with a change in wake mode from $2 \mathrm{~S}$, in the unsteady flow regime, to the $\mathrm{C}(\mathrm{AS})$ 

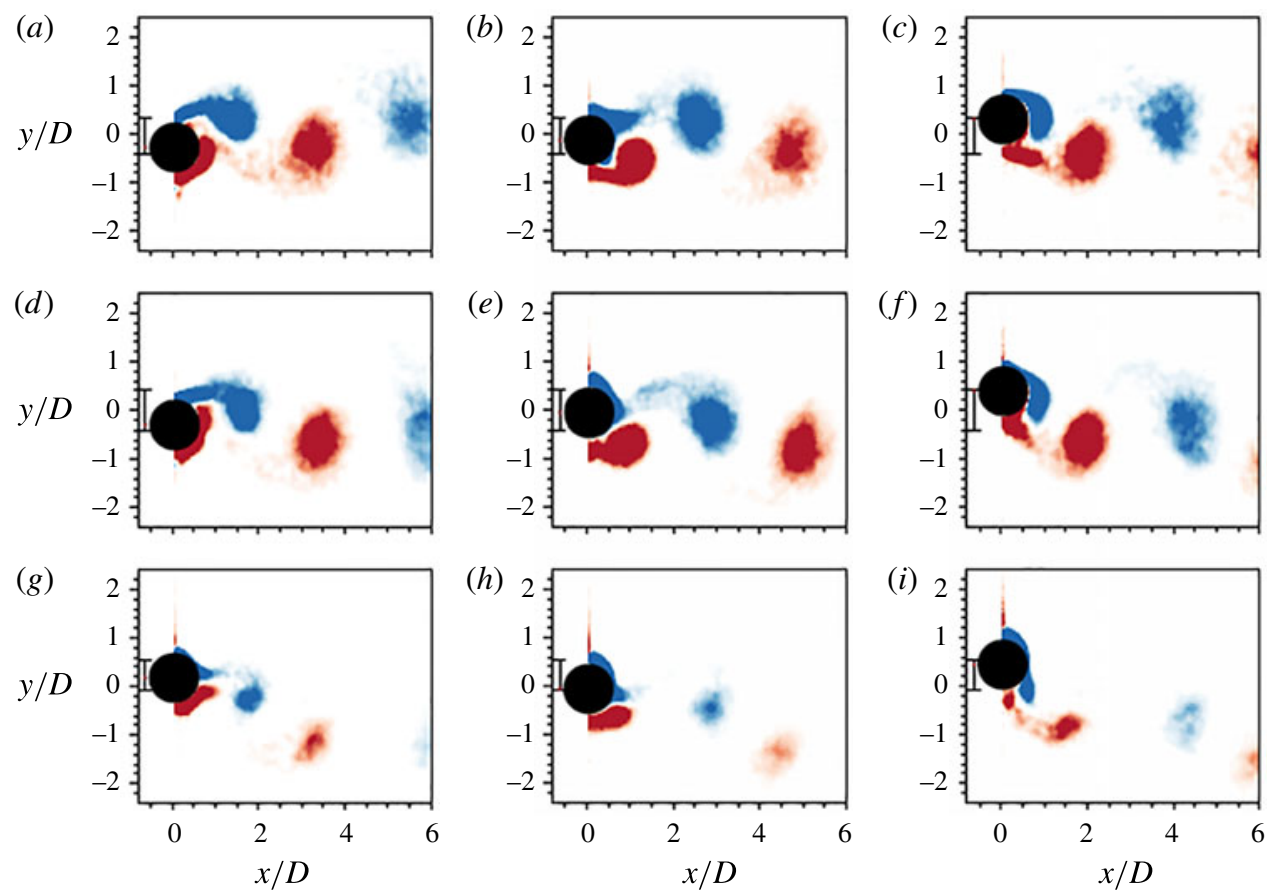

FIgURE 11. (Colour online) Phase-averaged spanwise vorticity contours of the $2 \mathrm{~S}$ mode at different $\alpha$ over an oscillation cycle: $U^{*}=5.00, \alpha=0.00(a-c), U^{*}=5.00, \alpha=1.00(d-f)$ and $U^{*}=5.00, \alpha=2.25(g-i)$. Contours were selected to align the leading vortex of each oscillation cycle, i.e. the vortex with positive vorticity, to the trailing vortex of the previous oscillation cycle. This illustrates the effects of $\alpha$ on wake asymmetry and the gap between vortices from adjacent oscillation cycles. Therefore, the contours between different $\alpha$ are not necessarily in phase. The normalised vorticity range is $\omega^{*}=\omega D / U \in[-1,1]$.

mode, where the flow becomes almost steady. At the start of the lower branch $\left(U^{*}=\right.$ 6.5) there is a similar wake transition when $P+S$ undergoes transition to $C(A S)$.

To further understand and characterise the effects of increasing rotation rate on the cylinder wake, the induced changes to the dominant $2 \mathrm{~S}$ mode are examined. Figure 11 shows the progression of the $2 \mathrm{~S}$ wake mode with increasing rotation rate at a constant reduced velocity of $U^{*}=5.0$. In figure 11, $(a-c)$ shows the characteristic vortex shedding for the non-rotating cylinder. Figure $11(d-f)$ and $(g-i)$ show shedding at non-zero rotation rates: $\alpha=1$ and 2.25, respectively. With increasing rotation rate, the mean position of the cylinder shifts upwards and the shed vortices deviate further downwards from the streamwise centreline as they advect downstream. This is expected as the body rotation causes wake deflection due to the Magnus effect.

Increased rotation also changes the spacing between the clockwise and anticlockwise vortex cores in the wake. With no rotation, the positive and negative vortex cores are distributed evenly along the wake. However, the addition of rotation means that the wake vortices show signs of collecting in pairs. This effect is enhanced with increasing rotation rate. This is shown in figure $11(c, f, i$. It can be seen that, as the rotation rate is increased, the distance between a shed anticlockwise (red) vortex and the subsequently formed clockwise (blue) vortex is less than the distance between that vortex and the anticlockwise vortex shed next. With increasing rotation rate, the size and strength 

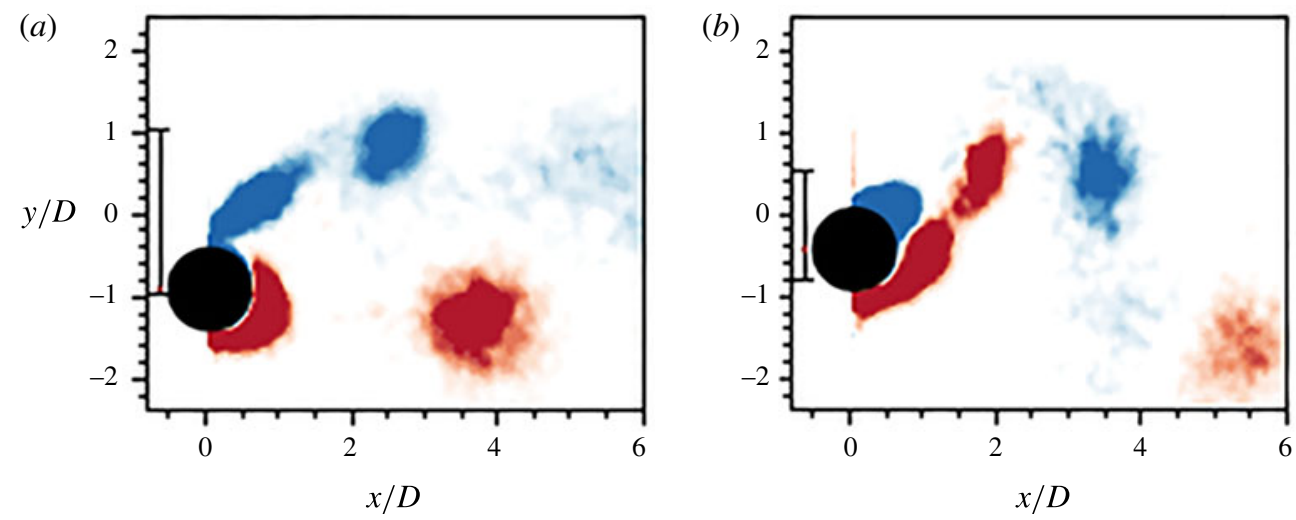

FIGURE 12. (Colour online) Phase-averaged isocontours of the $\mathrm{P}+\mathrm{S}$ mode at $U^{*}=$ 5.50, $\alpha=1.15$ in the upper response branch $(a)$ and $U^{*}=6.50, \alpha=0.60$ in the lower response branch $(b)$. The normalised vorticity range is $\omega^{*}=\omega D / U \in[-1,1]$.

of shed vortices decreases. This has been seen in rotating cylinder wakes previously, e.g. figure 5 from Radi et al. (2013). This is consistent with rotation causing the two separation points, which feed the shed vortices, to move together, thereby restricting the vorticity shed into each wake vortex and increasing cross-annihilation. Similar observations of the effects on the asymmetry and spacing are seen with the $\mathrm{P}+\mathrm{S}$ mode.

Images of the $\mathrm{P}+\mathrm{S}$ mode at different reduced velocities show a subtle change in the vortex pattern. Previous observations of the $\mathrm{P}+\mathrm{S}$ mode in VIV studies showed that it consisted of a single vortex on one side of the cylinder centreline, together with a clockwise and anticlockwise vortex pair on the other. Owing to symmetry in the non-rotating case, the pair and single vortices can form interchangeably on either side of the cylinder centreline. Figure 12 illustrates the $\mathrm{P}+\mathrm{S}$ mode in the upper amplitude response branch at $U^{*}=5.50$ (figure 12a) and in the lower response branch at $U^{*}=6.5$ (figure $12 b$ ). In the upper branch there are two clockwise vortices on the retreating side of the cylinder and one anticlockwise vortex on the advancing side of the cylinder. In the lower branch, the anticlockwise vortices on the advancing side of the cylinder that were previously observed in the upper branch remain. However, on the retreating side there are now one clockwise and one anticlockwise vortex. This observation was unexpected. It had been thought that the flow asymmetry created by the cylinder rotation would promote the shedding of vortices in one direction, hence the consistent combination of vortices seen in previous work (Williamson \& Roshko 1988; Morse \& Williamson 2009) and figure 12(b). This result was observed at a range of rotation rates in the upper and lower branch. Further work is needed to better understand this behaviour.

\subsection{Intermittent nature of the wake in the upper branch}

When a cylinder undergoes VIV, previous studies have shown that, in the upper branch, the wake is of an intermittent or chaotic nature (Lucor \& Triantafyllou 2008; Zhao et al. 2014a). One question of interest is whether this mode-switching signature of the upper branch still prevails when the cylinder is undergoing forced vibration. Indeed, the action of rotating fixed cylinders modifies the wake stability, whether 

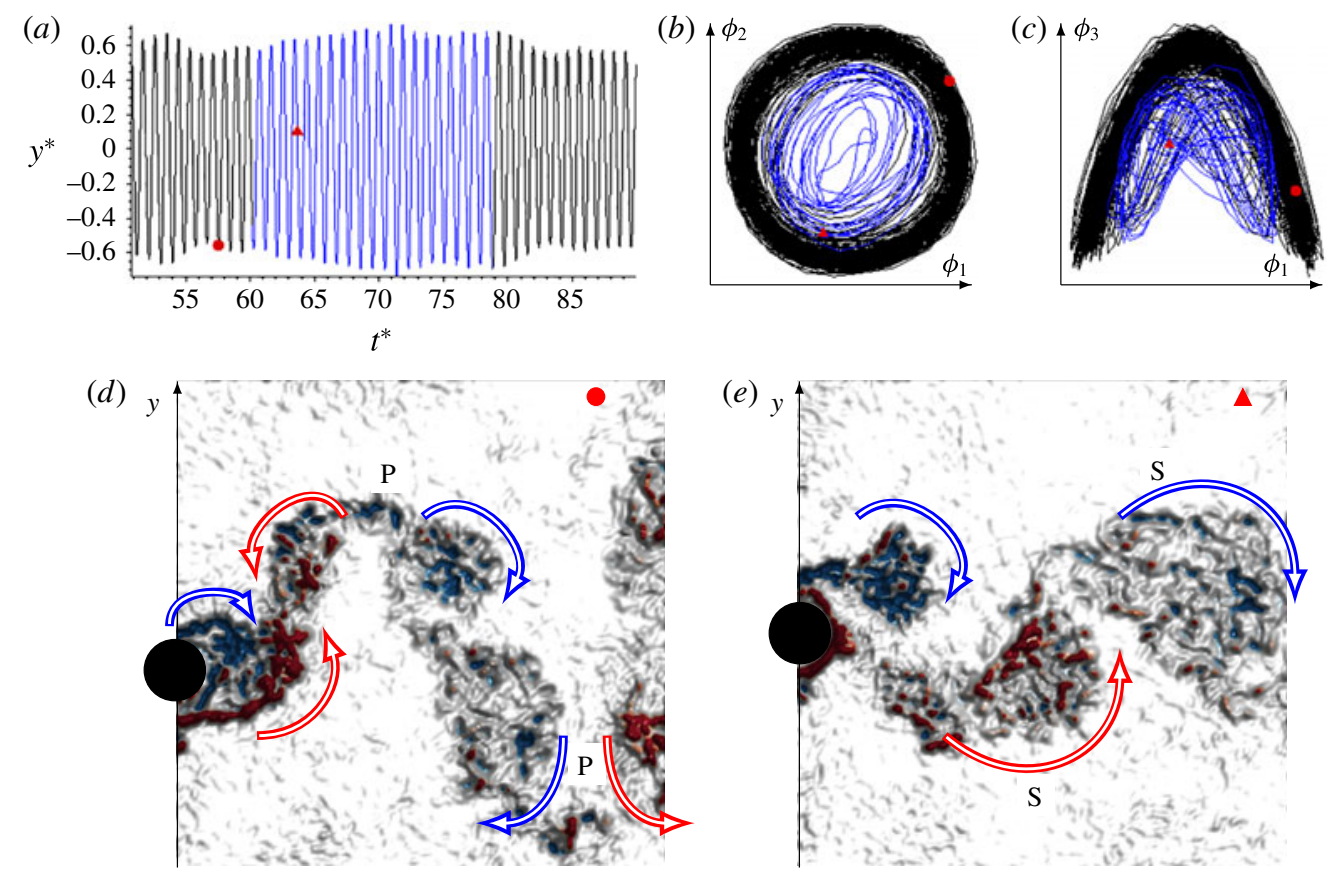

FIGURE 13. (Colour online) Instantaneous measurement showing transition from the $2 \mathrm{P}$ to $2 \mathrm{~S}$ mode for $U^{*}=6.50$ and $\alpha=0.0$. (a) Time-varying body position $y^{*}=y^{\prime} / D$, with $y^{\prime}=y-\bar{y}$, as a function of $t^{*}=t f_{n w}$. The black line represents the zone of $2 \mathrm{P}$ mode, and the blue line represents the zone of $2 \mathrm{~S}$ mode. The red filled symbols $(\boldsymbol{\bullet}$ and $\mathbf{\Delta})$ match the instantaneous vorticity fields presented in $(d)$ and $(e)$ showing a $2 \mathrm{P}$ and $2 \mathrm{~S}$ mode, respectively, to the instantaneous positions in the other plots. Plots $(b)$ and $(c)$ represent the temporal evolution of the POD modes, highlighting the differences over time (i.e. providing different Lissajous shapes). See supplementary movie 1.

the rotation is oscillatory (Tokumaru \& Dimotakis 1991; Lo Jacono et al. 2010; D'Adamo, Godoy-Diana \& Wesfreid 2015) or uniform (El Akoury et al. 2008; Rao et al. 2013, 2015). Thus, given that the cylinder is under uniform rotation, hence biasing the vorticity production towards one side and therefore biasing the wake topology, the question of how this affects the wake dynamics of the upper branch is of interest.

In the following, observations on the vortex wake state are presented for $U^{*}=6.5$ at three different rotation rates: $\alpha=0.0,0.8,1.5$. The switching of the wake state was identified from the inspection of the $\phi_{1}-\phi_{4}$ POD temporal coefficients. The instantaneous amplitude provided by $y(t)$ was not enough to unambiguously decipher changes within the wake, as exemplified through figures 13-15. Given the substantial number of snapshots gathered $(\sim 3100)$, covering a large number of shedding cycles $(\sim 200-300)$, it is possible to observe many examples of mode switching. In the supplementary material associated with this paper (available at https://doi.org/10.1017/jfm.2017.540), movies displaying these transitions provide a useful adjunct to the still images presented in this section.

Figure 13 shows an example of this mode-switching behaviour for $\alpha=0$. This example shows the transition from a $2 \mathrm{P}$ to $2 \mathrm{~S}$ shedding mode. The time history of the motion is given in figure 13(a), with the section in blue marking where mode 

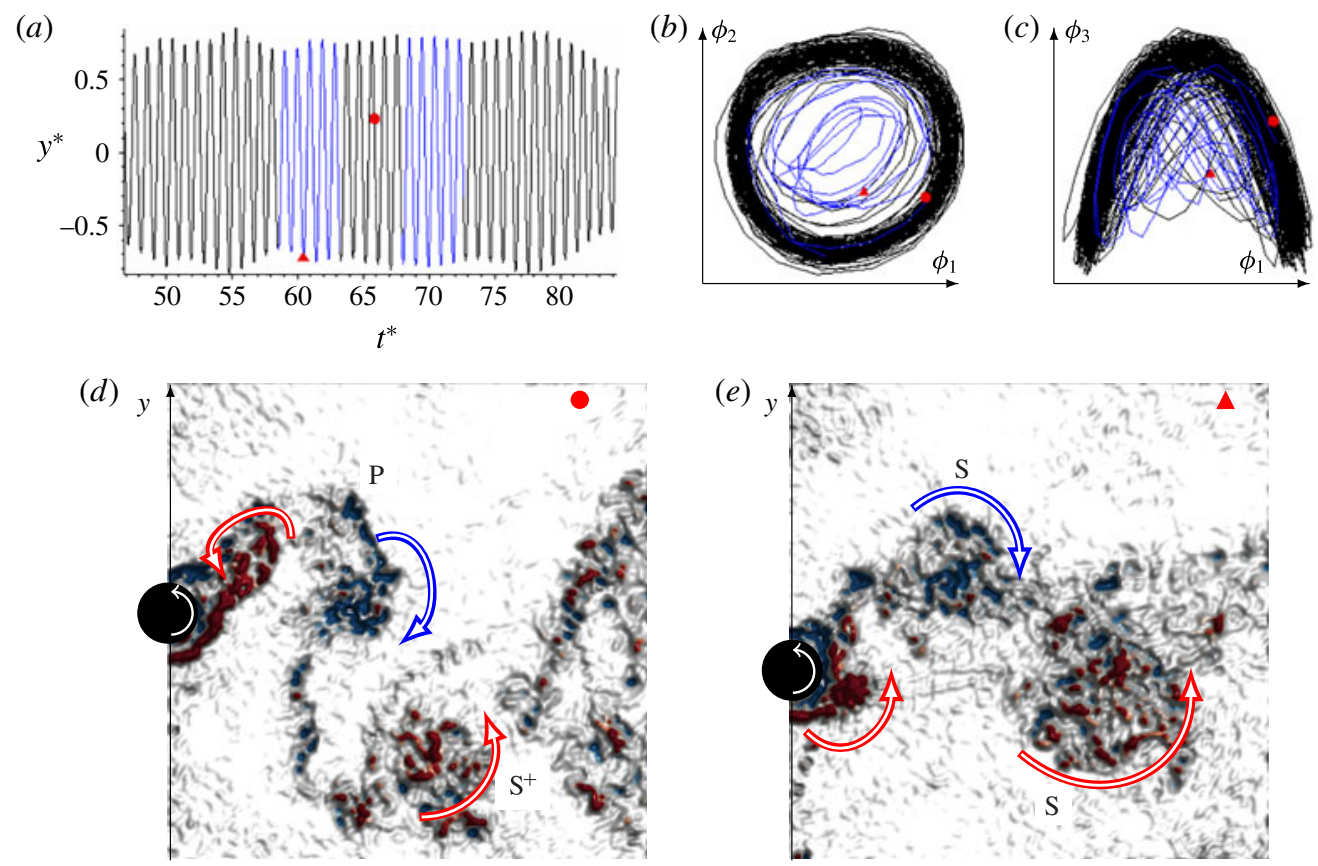

FIGURE 14. (Colour online) Instantaneous measurement showing transition from the $\mathrm{P}+$ $\mathrm{S}^{+}$to $2 \mathrm{~S}$ shedding mode for $U^{*}=6.50$ and $\alpha=0.80$. (a) Time-varying body position $y^{*}$ as a function of $t^{*}$. The black line represents the zone of $\mathrm{P}+\mathrm{S}^{+}$mode, and the blue line represents the zone of $2 \mathrm{~S}$ mode. The red filled symbols $(\boldsymbol{Q}$ and $\mathbf{\Delta})$ correspond to the instantaneous vorticity presented in panels $(d)$ and $(e)$ showing a $\mathrm{P}+\mathrm{S}^{+}$and $2 \mathrm{~S}$ mode, respectively. Plots $(b)$ and $(c)$ are representative of the temporal POD mode evolution, highlighting the differences in time (providing different Lissajous shapes). See supplementary movie 2 .

switching is observed. The temporal evolution of the mode is represented through Lissajous figures of the first pair $\left(\phi_{1}\right.$ and $\left.\phi_{2}\right)$, and the first and third modes $\left(\phi_{1}\right.$ and $\left.\phi_{3}\right)$, in figure $13(b, c)$, respectively. The symbols ( and $\left.\mathbf{\Delta}\right)$ appearing in these plots in figure $13(a-c)$ correspond to the instantaneous vorticity fields given in figure 13(d,e), representing the $2 \mathrm{P}$ and $2 \mathrm{~S}$ modes, respectively. Clearly, the information contained in the displacement signal $y(t)$ is not sufficient to reveal the behaviour within the wake, yet the changing form of the two Lissajous curves clearly differentiates the modes. In the supplementary movie, additional switching within the blue zone can be observed. Inspection of the POD temporal mode was instrumental to differentiate the wake dynamics and was only possible due to the large number of shedding periods recorded.

Figure 14 shows a further example of mode switching at $\alpha=0.80$. This example shows the transition from a $\mathrm{P}+\mathrm{S}^{+}$to $2 \mathrm{~S}$ shedding mode. Note that the $\mathrm{S}^{+}$represents an anticlockwise single vortex. The time history of the displacement trace is given in figure 14(a), with the mode switching zone coloured in blue. The temporal mode evolution is given through Lissajous figures of the first pair $\left(\phi_{1}\right.$ and $\left.\phi_{2}\right)$, and the first and third modes $\left(\phi_{1}\right.$ and $\left.\phi_{3}\right)$, in figure $14(b, c)$, respectively. The symbols appearing in figure $14(a-c)$ correspond to the instantaneous vorticity field given in figure $14(d, e)$, representing the $\mathrm{P}+\mathrm{S}^{+}$and $2 \mathrm{~S}$ modes, respectively. Again, the changing shape of the 

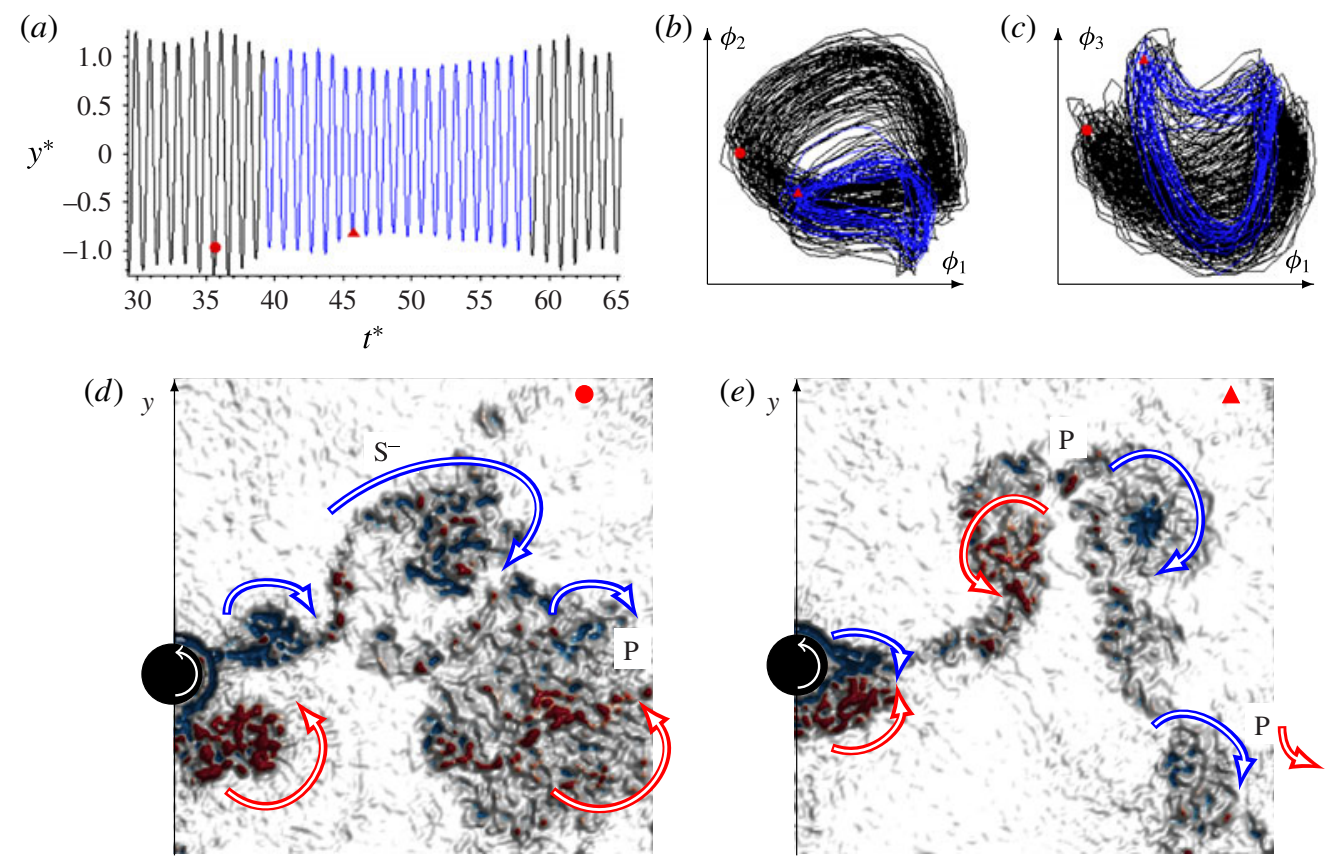

FIGURE 15. (Colour online) Instantaneous measurement showing transition from the $\mathrm{P}+$ $\mathrm{S}^{-}$to $2 \mathrm{~S}$ shedding mode for $U^{*}=6.50$ and $\alpha=1.50$. (a) Time-varying body position $y^{*}$ as a function of $t^{*}$. The black line represents the zone of $\mathrm{P}+\mathrm{S}^{-}$mode, and the blue line represents the zone of $2 \mathrm{~S}$ mode. The red filled symbols ( $\bullet$ and $\mathbf{\Delta})$ correspond to the instantaneous vorticity presented in panels $(d)$ and $(e)$, showing a $\mathrm{P}+\mathrm{S}^{-}$and 2P mode respectively. Plots $(b)$ and $(c)$ are representative of the temporal POD mode evolution, highlighting the differences in time (providing different Lissajous shapes). See supplementary movie 3 .

two Lissajous curves clearly differentiates the shedding modes, while the displacement signal $y(t)$ is not sufficient to reveal the mode switch.

As the rotation rate is increased to $\alpha=1.50$, the mode switching behaviour still prevails at $U^{*}=6.5$. Figure 15 shows the transition from a $\mathrm{P}+\mathrm{S}^{-}$to $2 \mathrm{P}$ mode. Note that $\mathrm{S}^{-}$represents a clockwise single vortex. As before, the time history of the motion is given, with the switch to blue marking the transition between wake states. Once again, the switch is clear through the change in the response depicted in the Lissajous figures (figure 15b,c). The supplementary movie 3 further confirms these modes, noting that it can be difficult to interpret a shedding mode from a single instantaneous snapshot.

It is acknowledged that the results presented do not provide a complete picture reflecting all possible transitions. However, the limited information reported is sufficient to confirm that the intermittent behaviour in the upper branch is sustained even for high rotation rates.

\subsection{Discussion}

Figure 16(a) shows a comparison of the vibration response curve at $\alpha=2$ with previous parameter studies from the literature. The response curve of SM15, who also performed experiments using a water channel for their VIV studies of a rotating 

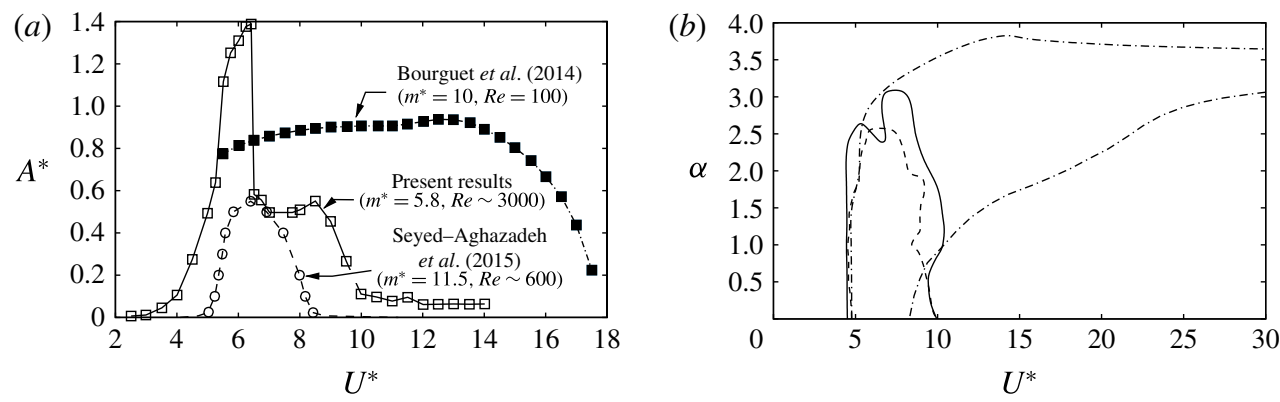

FIgURE 16. (a) Comparison of the response curve for $\alpha=2.0$ against previous numerical and experimental studies. Note, in particular, the much higher vibration response in this case. (b) Corresponding approximate vibration boundaries as a function of $U^{*}$ and $\alpha$. Refer to panel $(a)$ for data references.

cylinder, has been extracted from an amplitude contour plot in their paper. Their Reynolds-number range covers the interval $350 \leqslant R e<1000$, with the maximum response recorded for $U^{*}=6.5$, corresponding to $R e \simeq 600$. The $R e=100$ numerical results of BL14 are also shown for this rotation rate. The maximum response $\left(A^{*} \simeq 0.6 D\right)$ found by SM15 actually occurred at a slightly higher rotation rate of $\alpha \simeq 2.3$, although this was only marginally in excess of the value recorded for the $\alpha=2.0$ case shown. On the other hand, the low-Reynolds-number simulations of BL14 indicate that the vibration response continues to increase with rotation rate up to $\alpha=4.0$, after which there is minimal response. Notably, they also found a significant vibration response for a much larger $U^{*}$ range than observed in both sets of experiments. The different vibration response seen in the current experiments is likely to be due to the significantly higher Reynolds number $(R e=3000$ at $\left.U^{*}=6.5\right)$ of the current study, rather than the different mass ratios $\left(m^{*}=5.78\right.$ compared with 11.5 and 10), or the different but low mass-damping ratios, of the different experiments and simulations. Figure 16(b) shows the approximate vibration response boundaries for these three cases, delimiting regions where the response is non-negligible. Interestingly, the two sets of experiments show similar boundaries, despite the distinct differences in amplitude response. The unstable region from the numerical simulations is also similar over the initial $U^{*}$ range for low $\alpha$ but shows that significant vibration response extends up to high $U^{*}$ for larger values of $\alpha$.

\section{Conclusions}

The present study on the vibration response and wake structure of an elastically mounted circular cylinder subjected to forced, constant rotation over a range of rotation rates and Reynolds numbers has made several new findings.

Substantial body vibration was found to occur through wake-body synchronisation over a range of rotation rates and reduced velocities. Significant cylinder oscillations were observed to occur for rotation rates up to $\alpha \approx 3.5$ over the lock-in range. Interestingly, previous rigidly mounted cylinder studies show that rotation rates beyond $\alpha=2$ cause suppression of vortex shedding. Overall, this study shows that forced rotation can cause the cylinder to vibrate at up to $1.4 D$, a $76 \%$ increase in amplitude response over non-rotating VIV. This maximal response occurs when the rotation rate is close to 2.0 and in the range of reduced velocities associated with the upper 
amplitude response branch of a non-rotating cylinder. Across the $U^{*}-\alpha$ parameter space where a large amplitude response is seen, the cylinder frequency response is observed to be synchronised with the natural frequency of the oscillating system. It is the resonance between these two frequencies that enables large vortex-induced oscillations for non-rotating VIV and also enables large oscillations for VIV of a rotating cylinder. Increasing the rotation rate from 0 to 2 both broadens the range of reduced velocities where large body oscillations occur and increases the peak amplitude response. As the rotation rate is increased towards $\alpha \approx 2.0$, the frequency response $f^{*}$ gradually decreases to fall below $f^{*}=1$, where the oscillation frequency equals the natural frequency of the structure. This is correlated with the amplitude response increasing as the rotation rate is increased towards $\alpha \approx 2.0$. An increase in rotation rate beyond $\alpha \approx 2.0$ results in a reduced peak amplitude response, noting that the body oscillation becomes desynchronised with the weakened, less coherent vortex shedding.

A variety of wake modes were observed over the parameter space covered by the present study. At low to moderate reduced velocities $\left(U^{*}<6.5\right)$, the $2 \mathrm{~S}$ mode dominates at all rotation rates observed. At low rotation rates $(\alpha<1.25)$, the $2 \mathrm{~S}$ and $2 \mathrm{P}$ modes characterise the wake at reduced velocities associated with the upper and lower response branches, respectively. In the desynchronised region, the $2 \mathrm{~S}$ mode appears again. At moderate rotation rates $(1.25 \leqslant \alpha<2.25)$, the $\mathrm{P}+\mathrm{S}$ mode, a mode composed of a pair of counter-rotating vortices and a single vortex shed per cycle, was observed for most of the synchronisation region. Chaotic switching of the wake mode was observed in the upper branch. Indeed, it was found that, regardless of the rotation rate, the upper response branch seemed to retain its chaotic state. In the desynchronised region, a mode in which small-scale vortices coalesce and shed asymmetrically, $\mathrm{C}(\mathrm{AS})$, was observed at rotation rates above $\alpha \approx 1.25$. Beyond $\alpha \approx 2.25, \mathrm{C}(\mathrm{AS})$ dominates in the reduced velocity range associated with the vibration region of a non-rotating cylinder $\left(U^{*} \approx 4-10\right)$. On further increasing the rotation rate, shed vortices increasingly deviate from the streamwise centreline of the cylinder. This deviation with rotation rate was observed for multiple wake modes, as is expected from the Magnus effect. In addition, the distance between vortices of adjacent oscillation cycles increases, and the size and peak vorticity of the vortices shed decreases, in line with non-VIV studies.

Of further interest is the significantly 1 ower a mplitude $\mathrm{r}$ esponse o bserved experimentally by Seyed-Aghazadeh \& Modarres-Sadeghi (2015) relative to that found in this case. Given the substantial Reynolds-number difference between these studies, this could simply be a low-Reynolds-number effect, but it would be useful to have independent verification o f e ither s tudy. I $\mathrm{n}$ a $\mathrm{s}$ ense, $\mathrm{i}$ i $\mathrm{s} \mathrm{s}$ urprising $\mathrm{t}$ hat rotation significantly e nhances $\mathrm{v}$ ibration a mplitudes, $\mathrm{g}$ iven $\mathrm{t}$ hat $\mathrm{i} \mathrm{t} \mathrm{t}$ ends $\mathrm{t} \mathrm{o} \mathrm{m}$ ake $\mathrm{t}$ he wake narrower and asymmetric, so a deeper understanding of the near-wake dynamics and the effects on VIV would be useful. Also of interest is the still significant amplitude response observed at $\alpha=2.5$ and 3, well beyond the rotation rate for suppressing shedding from a fixed $r$ otating c ylinder, w hich $\mathrm{w}$ arrants $\mathrm{f}$ urther investigation.

\section{Acknowledgements}

The authors acknowledge financial s upport $\mathrm{f}$ rom $\mathrm{A}$ ustralian $\mathrm{R}$ esearch Council Discovery Project grants DP110102141 and DP150102879. The authors would like to acknowledge the financial s upport of the $\mathrm{C}$ entre $\mathrm{N}$ ational de la R echerche Scientifique (CNRS) through grant no. PICS161793 under the Projet International de Coopération Scientifique. K.W.L. a cknowledges fi nancial su pport from a Co mmonwealth sponsored Australian Postgraduate Award (APA) for his PhD study. 
Supplementary movies are available at https://doi.org/10.1017/jfm.2017.540.

\section{REFERENCES}

Badr, H. M., Coutanceau, M., Dennis, S. C. R. \& Ménard, C. 1990 Unsteady flow past a rotating circular cylinder at Reynolds numbers $10^{3}$ and $10^{4}$. J. Fluid Mech. 220, 459-484.

Bearman, P. W. 1984 Vortex shedding from oscillating bluff bodies. Annu. Rev. Fluid Mech. 16 (1), 195-222.

BeARman, P. W. 2011 Circular cylinder wakes and vortex-induced vibrations. J. Fluids Struct. 27 (5-6), 648-658.

Bearman, P. W., Gartshore, I. S., Maull, D. \& Parkinson, G. V. 1987 Experiments on flow-induced vibration of a square-section cylinder. J. Fluids Struct. 1 (1), 19-34.

Bishop, R. E. D. \& HASSAN, A. Y. 1964 The lift and drag forces on a circular cylinder in a flowing fluid. Proc. R. Soc. Lond. A 277, 32-50.

Blackburn, H. M. \& Henderson, R. D. 1999 A study of two-dimensional flow past an oscillating cylinder. J. Fluid Mech. 385, 255-286.

Blevins, R. D. 1990 Flow-Induced Vibration, 2nd edn. Krieger.

Bourguet, R. \& Lo JaCono, D. 2014 Flow-induced vibrations of a rotating cylinder. J. Fluid Mech. 740, 342-380.

Carberry, J., Sheridan, J. \& Rockwell, D. 2001 Forces and wake modes of an oscillating cylinder. J. Fluids Struct. 15 (1), 523-532.

Corless, R. M. \& PARkinson, G. V. 1988 A model of the combined effects of vortex-induced oscillation and galloping. J. Fluids Struct. 2 (3), 203-220.

CoutanceAu, M. \& MÉnARD, C. 1985 Influence of rotation on the near-wake development behind an impulsively started circular cylinder. J. Fluid Mech. 158, 399-446.

D’Adamo, J., Godoy-Diana, R. \& Wesfreid, J. E. 2015 Centrifugal instability of Stokes layers in crossflow: the case of a forced cylinder wake. Proc. R. Soc. Lond. A 471, 20150011.

Dahl, J., Hover, F., Triantafyllou, M., Dong, S. \& Karniadakis, G. 2007 Resonant vibrations of bluff bodies cause multivortex shedding and high frequency forces. Phys. Rev. Lett. 99 (14), 144503.

Den Hartog, J. P. 1932 Transmission line vibration due to sleet. Trans. Am. Inst. Electr. Engrs 51 (4), 1074-1076.

El Akoury, R., Braza, M., Perrin, R., Harran, G. \& Hoarau, Y. 2008 The three-dimensional transition in the flow around a rotating cylinder. J. Fluid Mech. 607, 1-11.

FENG, C. C. 1968 The measurement of vortex induced effects in flow past stationary and oscillating circular and D-section cylinders, Masters thesis, University of British Columbia.

Fouras, A., Lo Jacono, D. \& Hourigan, K. 2008 Target-free stereo PIV: a novel technique with inherent error estimation and improved accuracy. Exp. Fluids 44 (2), 317-329.

Govardhan, R. \& Williamson, C. H. K. 2000 Modes of vortex formation and frequency response of a freely vibrating cylinder. J. Fluid Mech. 420, 85-130.

Griffin, O. M., Skop, R. A. \& Koopmann, G. H. 1973 The vortex-excited resonant vibrations of circular cylinders. J. Sound Vib. 31 (2), 235-249.

He, J.-W., Glowinski, R., Metcalfe, R., Nordlander, A. \& PeriauX, J. 2000 Active control and drag optimization for flow past a circular cylinder. J. Comput. Phys. 163, 83-117.

Holmes, P., Lumley, J. L., Berkooz, G. \& Rowley, C. W. 2012 Turbulence, Coherent Structures, Dynamical Systems and Symmetry, Cambridge Monographs on Mechanics. Cambridge University Press.

Jauvtis, N. \& Williamson, C. H. K. 2004 The effect of two degrees of freedom on vortex-induced vibration at low mass and damping. J. Fluid Mech. 509, 23-62.

Kang, S., Choi, H. \& LeE, S. 1999 Laminar flow past a rotating circular cylinder. Phys. Fluids 11 (11), 3312-3321. 
Khalak, A. \& Williamson, C. H. K. 1997 Fluid forces and dynamics of a hydroelastic structure with very low mass and damping. J. Fluids Struct. 11, 973-982.

Khalak, A. \& Williamson, C. H. K. 1999 Motions, forces and mode transitions in vortex-induced vibrations at low mass-damping. J. Fluids Struct. 13, 813-851.

Legrand, M., Nogueira, J., Tachibana, S., Lecuona, A. \& Nauri, S. 2011 Flow temporal reconstruction from non time-resolved data. Part II: Practical implementation, methodology validation, and applications. Exp. Fluids 51, 861-870.

Lo Jacono, D., Leontini, J. S., Thompson, M. C. \& Sheridan, J. 2010 Modification of three-dimensional transition in the wake of a rotationally oscillating cylinder. J. Fluid Mech. 643, 349-362.

LuCor, D. \& Triantafyllou, M. S. 2008 Parametric study of a two degree-of-freedom cylinder subject to vortex-induced vibrations. J. Fluids Struct. 24, 1284-1293.

Mittal, S. \& Kumar, B. 2003 Flow past a rotating cylinder. J. Fluid Mech. 476, 303-334.

Morse, T. L. \& Williamson, C. H. K. 2009 Prediction of vortex-induced vibration response by employing controlled motion. J. Fluid Mech. 634, 5-39.

NaUdascher, E. \& RockWell, D. 2005 Flow-Induced Vibrations: An Engineering Guide. Dover.

Nemes, A., Zhao, J., Lo Jacono, D. \& Sheridan, J. 2012 The interaction between flow-induced vibration mechanisms of a square cylinder with varying angles of attack. J. Fluid Mech. 710, 102-130.

Païdoussis, M., Price, S. \& De LAngre, E. 2010 Fluid-Structure Interactions: Cross-Flow-Induced Instabilities. Cambridge University Press.

Pralits, J. O., Brandt, L. \& Giannetti, F. 2010 Instability and sensitivity of the flow around a rotating circular cylinder. J. Fluid Mech. 650, 513.

Pralits, J. O., GiannetTi, F. \& BrandT, L. 2013 Three-dimensional instability of the flow around a rotating circular cylinder. J. Fluid Mech. 730, 5-18.

Radi, A., Thompson, M. C., RaO, A., Hourigan, K. \& Sheridan, J. 2013 Experimental evidence of new three-dimensional modes in the wake of a rotating cylinder. J. Fluid Mech. 734, 567-594.

Rao, A., Leontini, J., Thompson, M. C. \& Hourigan, K. 2013 Three-dimensionality in the wake of a rotating cylinder in a uniform flow. J. Fluid Mech. 717, 1-29.

Rao, A., Radi, A., Leontini, J. S., Thompson, M. C., Sheridan, J. \& Hourigan, K. 2015 A review of rotating cylinder wake transitions. J. Fluids Struct. 53, 2-14.

SARPKAYA, T. 2004 A critical review of the intrinsic nature of vortex-induced vibrations. J. Fluids Struct. 19 (4), 389-447.

Seyed-Aghazadeh, B. \& Modarres-Sadeghi, Y. 2015 An experimental investigation of vortexinduced vibration of a rotating circular cylinder in the crossflow direction. Phys. Fluids 27 (6), 067101.

Sherry, M., Nemes, A., Lo Jacono, D., Blackburn, H. M. \& Sheridan, J. 2013 The interaction of helical tip and root vortices in a wind turbine wake. Phys. Fluids 25 (11), 117102.

SiROVICH, L. 1987 Turbulence and the dynamics of coherent structures. I. Coherent structures. $Q$. Appl. Maths 45 (3), 561-571.

Stojković, D., Breuer, M. \& Durst, F. 2002 Effect of high rotation rates on the laminar flow around a circular cylinder. Phys. Fluids 14 (9), 3160-3178.

Stojković, D., Schön, P., Breuer, M.\& Durst, F. 2003 On the new vortex shedding mode past a rotating circular cylinder. Phys. Fluids 15 (5), 1257-1260.

SWANSON, W. M. 1961 The magnus effect: a summary of investigations to date. Trans. ASME J. Basic Engng 83 (3), 461.

Tietjens, O. K. G. \& Prandth, L. 1957 Rotating cylinder and magnus effect. In Applied Hydroand Aeromechanics: Based on Lectures of L. Prandtl, pp. 82-85. Dover.

Tokumaru, P. T. \& Dimotakis, P. E. 1991 Rotary oscillation control of a cylinder wake. J. Fluid Mech. 224 (1), 77-90.

Venning, J., Lo Jacono, D., Burton, D., Thompson, M. C. \& Sheridan, J. 2015 The effect of aspect ratio on the wake of the Ahmed body. Exp. Fluids 56 (6), 126. 
Williamson, C. H. K. \& Govardhan, R. 2004 Vortex-induced vibrations. Annu. Rev. Fluid Mech. 36 (1), 413-455.

Williamson, C. H. K. \& Roshko, A. 1988 Vortex formation in the wake of an oscillating cylinder. J. Fluids Struct. 2 (4), 355-381.

XIAO, Q. \& ZHU, Q. 2014 A review on flow energy harvesters based on flapping foils. J. Fluids Struct. 46, 174-191.

Young, J., Lai, J. C. \& Platzer, M. F. 2014 A review of progress and challenges in flapping foil power generation. Prog. Aerosp. Sci. 67, 2-28.

Zhao, J., Leontini, J. S., Lo JaCono, D. \& Sheridan, J. $2014 a$ Chaotic vortex induced vibrations. Phys. Fluids 26 (12), 121702.

Zhao, J., Leontini, J. S., Lo Jacono, D. \& Sheridan, J. $2014 b$ Fluid-structure interaction of a square cylinder at different angles of attack. J. Fluid Mech. 747, 688-721.

Zhao, M., Cheng, L. \& LU, L. 2014c Vortex induced vibrations of a rotating circular cylinder at low Reynolds number. Phys. Fluids 26 (7), 073602. 